\title{
An Image Denoising Method Based on BP Neural Network Optimized by Improved Whale Optimization Algorithm
}

\section{Chunzhi Wang}

School of Computer Science, Hubei University of Technology

\section{Min Li}

School of Computer Science, Hubei University of Technology https://orcid.org/0000-0001-9329-1086

\section{Ruoxi Wang}

Wuhan Fiberhome Technical Services Co.,Ltd.

\section{Han Yu}

Fiberhome Telecommunication Technologies Co.,Ltd.

\section{Shuping Wang ( $\sim$ wsping52@126.com)}

Huazhong University of Science and Technology https://orcid.org/0000-0002-4909-8649

\section{Research}

Keywords: whale optimization algorithm, nonlinear convergence factor, adaptive weight coefficient, BP neural network, image denoising

Posted Date: January 8th, 2021

DOl: https://doi.org/10.21203/rs.3.rs-137201/v1

License: (c) (1) This work is licensed under a Creative Commons Attribution 4.0 International License. Read Full License 


\title{
RESEARCH
}

\section{An Image Denoising Method Based on BP Neural Network Optimized by Improved Whale Optimization Algorithm}

\author{
Chunzhi Wang ${ }^{1}$, Min $\mathrm{Li}^{1}$, Ruoxi Wang ${ }^{2}$, Han $\mathrm{Yu}^{3}$ and Shuping Wang ${ }^{4 *}$
}

\begin{abstract}
As an important part of smart city construction, traffic image denoising has been studied widely. Image denoising technique can enhance the performance of segmentation and recognition model, and improve the accuracy of segmentation and recognition results. However, due to the different types of noise and the degree of noise pollution, the traditional image denoising methods generally have some problems, such as blurred edges and details, loss of image information. This paper presents an image denoising method based on BP neural network optimized by improved whale optimization algorithm. Firstly, the nonlinear convergence factor and adaptive weight coefficient are introduced into the algorithm to improve the optimization ability and convergence characteristics of the standard whale optimization algorithm. Then, the improved whale optimization algorithm is used to optimize the initial weight and threshold value of BP neural network to overcome the dependence in the construction process, and shorten the training time of the neural network. Finally, the optimized BP neural network is applied to benchmark image denoising and traffic image denoising. The experimental results show that compared with the traditional denoising methods such as Median filtering, Neighborhood average filtering and Wiener filtering, the proposed algorithm has better performance in peak signal-to-noise ratio (PSNR) than other algorithms.
\end{abstract}

Keywords: whale optimization algorithm; nonlinear convergence factor; adaptive weight coefficient; BP neural network; image denoising

\section{Introduction}

Smart city refers to the use of new generation of information technology to connect and integrate the city's systems and services, so as to improve the efficiency of resource utilization, optimize city services, achieve fine and dynamic management, and improve the quality of life of citizens[1]. From the perspective of technological development, smart city realizes comprehensive perception, ubiquitous interconnection, pervasive computing and converged application through the support of the new generation of information technology represented by the Internet of Things (IOT), mobile Internet, cloud computing and big data $[2,3,4]$. For example, relying on the IoT and mobile Internet can realize intelligent perception, identification, positioning, tracking and supervision while with the help of cloud computing, big data and intelligent analysis technology, massive information processing and decision support can be realized. 
Intelligent transportation system (ITS) is one of the important parts of smart city construction. Through monitoring, traffic flow distribution optimization and other technologies, the traffic information network system is improved and unified intelligent urban traffic integrated management and service system is established to realize the full sharing of traffic information, real-time monitoring and dynamic management $[5,6]$. ITS is an important technical means of modern transportation management in recent years. Its essence is a traffic system which uses sensors, network transmission, intelligent control, multimedia processing and other technologies to carry out large-scale, comprehensive, real-time and efficient monitoring and management $[7,8]$. ITS has brought huge economic and social benefits to the transportation industry and has become an important part of modern transportation system[9]. However, surveillance video and image acquisition in ITS are often limited by various factors such as acquisition equipment and external environment and there is a certain amount of noise, which affects the visibility and further processing of the image. Image denoising is one of the important technical means to improve the image visibility, and also one of the image preprocessing steps. It can provide a higher quality image source for further image processing.

Image denoising, as an image processing technology aiming at eliminating image noise, has attracted more and more attention from scholars. For image segmentation, image recognition and other technologies, image denoising can enhance the performance of segmentation and recognition model, and improve the accuracy of segmentation and recognition results[10,11]. The traditional image denoising methods are mainly divided into space domain denoising method and transform domain method according to the different denoising processing domain[12, 13, 14, 15]. Among them, the spatial domain denoising methods directly analyze and process the gray value of the pixels in the noised image, mainly including Mean filtering, Median filtering and Gaussian filtering. These methods can suppress the noise in the image to a certain extent, but because all the pixels in the image are processed in the same way, it also blurs the edges and details in the image while smoothing the noise, resulting in the loss of image information[16]. Transform domain denoising methods transform the noised image from the spatial domain to the corresponding transform domain by using different transformation methods, mainly including Fourier transform, Wavelet transform, sparse representation and denoising methods combined with non-local denoising theory, etc. These methods have greatly promoted the research of image denoising[17].

In recent years, neural network has been widely used in image feature extraction, image denoising, image segmentation and image recognition because of its good nonlinear mapping, parallel computing and adaptive ability[18]. In the research of various application fields of neural network image processing, about 80\% 90\% of the applications are back propagation (BP) neural network. But BP neural network uses gradient descent search algorithm, so it is sensitive to the initial weight threshold[19, 20], and the algorithm is easy to converge to the local minimum. To solve this problem, there are usually two kinds of improvement methods: the first is to use the additional momentum method, the adaptive learning rate method with momentum term and Levenberg-Marquard algorithm, etc., to improve the operation mechanism of the algorithm and improve the search ability of the algorithm. This 
kind of method can increase the running speed, but it can not solve the problem of falling into local minimum caused by the randomness of weight threshold. The second is to use swarm intelligence algorithms such as genetic algorithm, particle swarm optimization algorithm to determine the initial weight threshold of BP neural network, and then use Levenberg-Marquard algorithm for optimization, which can effectively reduce the possibility of BP neural network falling into local minimum.

Whale optimization algorithm (WOA)[21] is a new swarm intelligence optimization algorithm proposed by Seyedali Mirjalili in 2016 which simulating the behavior of whale predation. Compared with particle swarm optimization (PSO), gravitational search algorithm (GSA) and other classical intelligent optimization algorithms, this algorithm has the advantages of simple structure, less parameters and strong optimization ability[22, 23]. It has been widely concerned by many scholars and applied to different practical problems. However, like other intelligent optimization algorithms, the standard whale optimization algorithm also has the problems of slow convergence speed and easy to fall into local optimum.

\subsection{Our Contributions}

Based on the existing research results, this paper proposes an image denoising method based on BP neural network optimized by improved whale optimization algorithm. Compared with Median filtering(MF), Neighborhood averaging filtering(NAF), Wiener filtering(WF) and traditional BP neural network denoising algorithm, the peak signal-to-noise ratio (PSNR) of the image is significantly improved. To be specific, the major contributions of this paper are threefold:

- The nonlinear convergence factor and adaptive weight coefficient are introduced into the whale optimization algorithm to improve the standard whale optimization algorithm, which improves the optimization ability and convergence characteristics of the algorithm.

- The improved whale optimization algorithm is used to optimize the initial weights and thresholds of BP neural network, which overcomes the dependence on the initial weights and thresholds in the construction process of BP neural network, and shortens the training time of the neural network.

- The optimized BP neural network is applied to image denoising. The benchmark images and UAS data set are selected for denoising experiment. The results show that MSWOA-BP has better denoising effect than other denoising algorithms.

\subsection{Organization of the Rest Paper}

The rest of the paper is arranged as follows. Section 2 reviews traffic image denoising, the application of BP neural network in image denoising and the improvement of whale optimization algorithm. In section 3 , an improved whale optimization algorithm based on mixed strategy and an image denoising method based on improved BP neural network are proposed.In section 4, the complexity of the improved whale optimization algorithm is analyzed. In section 5, experiments are carried out to verify the effectiveness of the improved whale optimization algorithm, and then the proposed denoising method is compared with the traditional method to verify the effectiveness of the method. Finally, some concluding remarks are presented in section 6 . 


\section{Related Work}

Recently, traffic image denoising has been studied. In [24], a traffic image denoising method based on low rank decomposition is proposed. The experimental results show that the proposed algorithm can remove noise more effectively and retain the effective information of the image compared with BM3D, KSVD and PCA, and has better performance in the evaluation index PSNR and visual effect. [25] introduced the sparse representation method to traffic image processing, and realized a traffic image denoising algorithm based on K-SVD orthogonal matching pursuit. Experimental results show that compared with the traditional image enhancement methods (Median filtering, Mean filtering, Wavelet filtering) and sparse representation image enhancement method based on DCT redundant dictionary, the proposed algorithm can remove traffic image noise more effectively and get higher PSNR. Aiming at the problem of image denoising in license plate recognition preprocessing, an adaptive coupled partial differential equation (PDE) denoising model is proposed in [26], which can more effectively remove the mixed noise in the license plate image, protect the edge information of the image, and improve the PSNR. The denoised image is more conducive to the subsequent character segmentation and recognition. It can effectively improve the recognition accuracy of license plate image.

In order to solve the problems of BP neural network in the process of image denoising that the convergence speed is slow and it is easy to fall into the local extreme value, great efforts have been made to improve it.According to the distribution characteristics of speckle noise in medical image, Jing et al.[27] proposed a denoising model based on the nonlinear mapping ability of BP neural network. The model can remove the speckle noise in the ultrasonic image and retain the edge features of the image, but the whole model has poor denoising effect and long running time. Yan et al.[28] proposed a new image denoising method based on BP neural network improved by improved copula distribution estimation algorithm. The centroid mutation operator is introduced on the basis of traditional copula distribution estimation algorithm, which effectively overcomes the shortcoming that BP neural network is easy to fall into local optimum in image denoising, but the overall complexity of the model is high and the overall performance is poor. Aiming at the shortcomings of BP neural network in image denoising, such as slow convergence speed, easy to fall into local minimum and so on, Wang et al.[29] used gray wolf optimization algorithm to optimize the initial parameters of the neural network. The convergence speed of the improved neural network is greatly improved, but the denoising effect still needs to be improved. On the basis of [29], [30] improves the noise removal ability of the model through enhancing the global optimization ability of gray wolf optimization algorithm by changing the convergence mode of convergence factor and introducing differential evolution to increase population diversity. It has improved the denoising ability of the image polluted by Gaussian noise of the improved model, but it has not greatly improved the denoising ability of other noises.

In order to solve the problems of slow convergence speed, premature convergence and easy to fall into local optimum in the whale optimization algorithm, effective improvements have been made to the standard algorithm[31]. Kaur et al.[32] proposed CWOA by using chaotic map to optimize the update probability $p$ in WOA, it 
is verified that the algorithm has a high convergence speed through the benchmark function test. Mafarja et al.[33] fused simulated annealing algorithm and whale optimization algorithm to optimize the optimization accuracy of the algorithm and improve the global search capability. They also obtained good results in the public test of 18 data sets in the UCI library. Elaziz et al.[34] proposed OBWOA by using reverse learning for initialization that enhanced the algorithm to explore the search space, and applied OBWOA to three different diode models to estimate the parameters of the solar cell. Experiments showed that the method has good performance. Zhang et al.[35] proposed MOWA by first initializing the population by generating a chaotic sequence from the segmented logistic chaotic map to maintain the initial population diversity and introducing segmented adaptive weights at the same time to balance the algorithm's global exploration and local development capabilities. The performance of the algorithm was verified by testing on six benchmark functions. In order to solve large-scale complex optimization problems, Long et al.[36] first used the opposite learning strategy to initialize the population position, designed a nonlinear convergence factor, coordinated the exploration and development capabilities of the algorithm, and introduced diversity mutation operations to improve the algorithm's premature convergence. However, although the existing research has improved the optimization effect of the standard WOA, the problems such as the balance between global exploration and local development ability, slow convergence speed and easy to fall into local optimization still need to be further studied[37, 38, 39].

\section{Methodology}

3.1 Whale Optimization Algorithm And Its Improvement

\subsubsection{Whale Optimization Algorithm}

The inspiration of WOA originates from the unique foraging behavior of humpback whales that it follows a spiral-shaped path to forage by creating a bubble net after finding a foraging target(as shown in Fig.1). The specific behaviors include encircling prey, bubble-net attacking and search for prey[40]. Among them, the bubble-net attacking can be divided into shrinking encircling mechanism and spiral updating position. The foraging behavior of humpback whales can be described mathematically as follows:

Encircling Prey In the process of hunting, the whale needs to determine the position of its prey before encircling and capturing its prey. Since the position of prey in the search space is unknown, WOA assumes that the optimal solution in the current population is the target prey. After determining the prey, other whales in the population will update their positions according to the current position of the prey. In the process of predation, the following formula is used to update the position:

$$
\begin{aligned}
& D=\left|C \cdot X^{*}(t)-X(t)\right| \\
& X(t+1)=X^{*}(t)-A \cdot D
\end{aligned}
$$


where $t$ is the current number of iterations, $X^{*}(t)$ indicates the optimal whale's position in the $t$ th iteration. $X(t)$ is the position of the individual whale in the $t$ th iteration. $A$ and $C$ are coefficients calculated using the following equation:

$$
\begin{aligned}
& A=2 a \cdot r_{1}-a \\
& C=2 \cdot r_{2}
\end{aligned}
$$

where $r_{1}$ and $r_{2}$ are random numbers in [0,1], $a$ decreases linearly from 2 to 0 as the number of iterations increases.

Bubble-net attacking In WOA, the whale chooses one of the two behaviors of shrinking encircling and spiral updating position according to the random probability value. The shrinking encircling is realized by Eq.(3), the convergence factor $a$ decreases linearly as the number of iterations increases, and $A$ is a random value between $[-a, a]$. If $|A| \leq 1$, the individual whale gradually approaches the prey after updating its position, and completes the shrinking and encircling of the prey according to Eq.(2). The spiral updating position first needs to calculate the distance between the individual whale and the prey, and then prey on the prey in a spiral form. The mathematical model of this behavior is described as follows:

$$
\begin{aligned}
& D^{\prime}=\left|X^{*}(t)-A(t)\right| \\
& X(t+1)=X^{\prime} \cdot e^{b l} \cdot \cos (2 \pi l)+X^{*}(t)
\end{aligned}
$$

where Eq.(5) indicates the distance between the $i$ th whale and its prey. $l$ is a random number in $[-1,1]$, when $l=-1$, the individual whale is the closest to the foraging target, and when $l=1$, the individual whale is the farthest from the foraging target; $b$ is a helical constant used to define the shape of the logarithmic spiral.

In the process of bubble-net attacking, the behavior probability of whale choosing position updating mode is set as $p$. WOA assumes that the probability $p$ of performing these two kinds of predatory behavior is $50 \%$ respectively. The attacking process can be described as follows:

$$
X(t+1)= \begin{cases}X^{*}(t)-A \cdot D & p<0.5 \\ D^{\prime} \cdot e^{b l} \cdot \cos (2 \pi l)+X^{*}(t) & p>0.5\end{cases}
$$

Search for prey When $|A|>1$, WOA randomly selects an individual in the population to conduct random search. The mathematical model is as follows:

$$
\begin{aligned}
& D=\left|C \cdot X_{\text {rand }}-X\right| \\
& X(t+1)=X_{\text {rand }}-A \cdot D
\end{aligned}
$$

where $X_{\text {rand }}$ is the position of a random individual in the current population. 


\subsubsection{Improved Whale Optimization Algorithm Based on Mixed Strategy}

Although the standard whale optimization algorithm has few parameters and excellent performance, it still has the problems of low solution accuracy, slow convergence speed and easy to fall into local optimum. In order to overcome these shortcomings, this paper improves the algorithm from the two aspects of location update strategy and prevention of falling into local optimum, and proposes an improved whale optimization algorithm based on mixed strategy (MSWOA).

Nonlinear Convergence Factor Similar to other swarm intelligence optimization algorithms, whale optimization algorithm will have an imbalance between global search ability and local search ability in the process of finding the optimal solution.In the standard whale optimization algorithm, the parameter that controls whether the algorithm performs global search or local search is $A$. when $|A| \geq 1$, the algorithm performs random global search with a probability of 0.5 ; when $|A|<$ 1, the algorithm performs local search. The value of $A$ mainly depends on the convergence factor $a$. Therefore, the change of the convergence factor $a$ is very important for the algorithm to find the optimal solution. The larger convergence factor can provide strong global search ability and avoid the algorithm from falling into local optimum, while the smaller convergence factor can provide strong local search ability and accelerate the convergence speed of the algorithm. In the standard whale optimization algorithm, the convergence factor $a$ decreases linearly as the number of iterations increases. This change makes the convergence speed of the algorithm too slow. Therefore, inspired by the improvement strategy proposed in $[37,39,41]$, this paper introduces a nonlinear strategy to dynamically adjust the original convergence factor $a$ on the premise of not changing the overall change trend of the original convergence factor $a$, which can balance the global search ability and local search ability of the algorithm and accelerate the convergence speed of the algorithm. The mathematical formula is as follows:

$$
a=\frac{2}{e-1} \times\left(e^{1-\left(\frac{t}{T}\right)^{\lambda}}-1\right)
$$

where $t$ is the current number of iterations, $T$ is the maximum number of iterations, and $\lambda$ is a constant. In this paper, we take $\lambda=3$. The change of the improved convergence factor is shown in Fig.3.

Adaptive Weight Coefficient In the standard whale optimization algorithm, the position of the prey is the position of the optimal solution. However, during the execution of the algorithm, the position $X^{*}(t)$ of the prey in the position update Eqs.(2) (6) (9) is not fully utilized. In this paper, adaptive weight coefficient is introduced to use the optimal solution to improve the accuracy of the algorithm. The adaptive weight coefficient is defined as follows:

$$
\begin{aligned}
& \omega(t)=\frac{4}{\pi} \arctan \left(\frac{t}{\text { max_iter }}\right) \\
& X(t+1)=\omega(t) \cdot X^{*}(t)-A \cdot D \quad|A|<1, p<0.5
\end{aligned}
$$




$$
\begin{aligned}
& X(t+1)=\omega(t) \cdot X_{\text {rand }}-A \cdot D \quad|A| \geq 1, p<0.5 \\
& X(t+1)=D^{\prime} \cdot e^{b l} \cdot \cos (2 \pi l)+(1-\omega(t)) \cdot X^{*}(t) \quad p \geq 0.5
\end{aligned}
$$

where max_iter is the maximum number of iterations.

In Eqs.(12) (13), the adaptive weight coefficient $\omega(t)$ increases with the increase of iteration, which means that the position of prey is closer to the position of theoretical optimal solution after each iteration, so as to improve the optimization accuracy of the algorithm; in spiral updating, with the increase of iteration times, whales will continue to approach prey, and at this time, a smaller weight is used to facilitate the whale to better find whether there is a better solution around the prey while updating the position, thereby improving the local search ability of the algorithm .

\subsection{BP Neural Network}

BP neural network is a kind of neural network with signal forward propagation and error back-propagation. It is mainly composed of three neuron layers, namely input layer, hidden layer and output layer[42, 43]. The neurons between layers are all connected, and the neurons in the layer are not connected. The number of hidden layers is not fixed[44]. Take one hidden layer as an example, its structure is shown in Fig.2.

In the process of forward propagation, the input variables are transferred to the output layer through the input node in input layer and hidden layer, and the required information is obtained at the output layer. The weights and offsets of each network node should be consistent, and the neuron states of each layer are transmitted through activation function. If the error cannot be converged, then the error back propagation is carried out. In the process of error back propagation, contrary to forward propagation, the error is propagated layer by layer from the output layer to the input layer.By back propagating the output error, the error is allocated to all the cells of the original layer, so as to further obtain the error signal of each layer, and then correct the weight of each cell. Through repeated training, the weights and offsets of the nodes are modified until the expected error accuracy is achieved.

\subsection{Image Denoising Method Based on MSWOA-BP}

In this section, we propose a image denoising method based on BP neural network optimized by the improved whale optimization algorithm that proposed in section 3.1.2. It mainly includes three stages of network construction, network training and network testing.

Network Construction BP neural network can contain different hidden layers. However,it has been proved theoretically that three-layer BP neural network can approximate any continuous function. In addition, too many hidden layers will increase the complexity of the network and seriously reduce the computing speed of the network, so this paper uses a three-layer network structure in the research.

For the setting of network parameters, this paper uses the improved whale optimization algorithm in section 3.1.2 to optimize the BP neural network, mainly to 
optimize the initial weight and threshold of the BP neural network, so as to overcome the poor overall performance of the BP neural network due to its dependence on the initial value of the network, so as to achieve the purpose of optimizing the network model. The specific steps to obtain the initial weights and thresholds of the BP neural network by improved whale optimization algorithm are as follows:

Step1: Determine the topology of BP neural network, and set the number of nodes in the input layer, hidden layer, and output layer of the neural network (inputnum,hiddennum, outputnum ) and other related parameters;

Step2: Initialize $N$, Max_iter, $l b, u b$ and other parameters in whale optimization algorithm, and calculate the dimension dim according to the number of nodes in each layer of BP neural network set in step1. And then initialize the initial position $X_{i}=\left(x_{i 1}, x_{i 2}, \ldots, x_{i d i m}\right)^{T},(i=1,2, \ldots, N)$ of the whale population $X=\left(X_{1}, X_{2}, \ldots, X_{N}\right)$. The dimension $\operatorname{dim}$ is calculated as follows:

dim $=$ inputnum $*$ hiddennum + hiddennum + hiddennum $*$ outputnum + outputnum

Step3: Calculate and compare the fitness $f_{i}$ of each gray wolf individual in the population to determine the whale individual with the best current fitness value, defined as $X^{*}$. In this paper, the weight and threshold corresponding to the individual in the population are taken as the weight and threshold of BP neural network in each iteration, and the absolute error value of BP neural network is taken as its fitness value;

Step4: Update the position of every whale in the population. If $p<0.5$ and $|A|<1$, use Eq.(3), otherwise use Eq.(9). If $p \geq 0.5$, use Eq.(6);

Step5: Evaluate the whole whale population to find the global optimal whale and its positions;

Step6: If the termination condition (maximum number of iterations) of the algorithm is met, it ends; otherwise, go to Step3 and continue the algorithm iteration;

Step7: The global optimal solution $X^{*}$ obtained by whale optimization algorithm is transformed into the weight $(W 1, W 2)$ and threshold $(B 1, B 2)$ of BP neural network.

Network Training In image degradation, the gray value of a point is closely related to the gray value of its neighborhood. Even if the gray value of a pixel is the same, if its neighborhood is different, the gray value after degradation is not the same. In view of the great influence of the field on the gray value, this paper uses the idea of block when extracting image features. As shown in Fig.4, the noise picture is padded firstly, and then the gray value corresponding to each pixel and several pixels around it is used as the input of the neural network. The entire padded noised image is traversed using a sliding window to obtain all the input data of the neural network training. For the output data, the output of the model is the noiseless pixel point corresponding to the center of the sliding window. The determination of the input and output data also determines the number of nodes in the input layer and output layer of the BP neural network. 
Network Testing For the network model obtained in the previous stage, in order to test the denoising ability of the model, this paper selects common benchmark images for denoising experiments. By adding Gaussian noise of different intensities to the benchmark images, the noised images are obtained and used as the input of the model. Through the denoising model, the denoised images are obtained. The denoised images are compared with the standard images to obtain the peak signalto-noise ratio(PSNR).

\section{Complexity Analysis of Improved Algorithm}

The computational complexity depends on the number of executions of the algorithm. In the standard whale optimization algorithm, the computational complexity is mainly related to the population size $N$, the maximum number of iterations Max_iter and the search space dimension Dim, and the computational complexity is $O(N \cdot$ Max_iter $\cdot \operatorname{Dim})$. According to the process of the improved algorithm, the introduction of a non-linear convergence factor adjustment strategy and an adaptive weight coefficient increases the amount of operations for $O(N \cdot$ Max_iter $\cdot$ Dim $)$, and its computational complexity is $O\left(2 \cdot N \cdot M a x \_i t e r \cdot D i m\right)$, which is higher than that of the standard whale optimization algorithm.

The space complexity is mainly affected by the population size $N$ and the search space dimension Dim. Since the improved algorithm has no changes in the population size and search space dimension, the space complexity is $O(N \cdot \operatorname{Dim})$.

\section{Results and Discussion}

The simulation experiments in this paper are based on Intel(R) Core(TM) i57300HQ CPU, $2.50 \mathrm{GHz}, 16 \mathrm{~GB}$ memory and Windows 10 (64-bit) operating system, and the programming software is Matlab R2016b.

\subsection{Evaluation index of denoising effect}

In order to evaluate the denoising effect of the proposed denoising model, the peak signal-to-noise ratio (PSNR) is used to compare the denoised image with the standard image. PSNR is the ratio of peak signal energy to average noise signal energy. The unit is the logarithmic variable component $(\mathrm{dB})$. This evaluation method is based on mean square error (MSE), and the formula of mean square error of two gray-scale images $A(i, j)$ and $B(i, j)$ with size of $m \times n$ is shown in Eq.(16):

$$
M S E=\frac{1}{m n} \sum_{i=1}^{m} \sum_{j=1}^{n}\|A(i, j)-B(i, j)\|^{2}
$$

Based on MSE, the formula of PSNR is shown in Eq.(17):

$$
P S N R=10 \cdot \log _{10}\left(\frac{M A X_{I}^{2}}{M S E}\right)
$$

where $M A X_{I}$ is the maximum pixel value in the image. 


\subsection{The Performance of Improved Whale Optimization Algorithm}

In order to test the optimization ability of the improved whale optimization algorithm (MSWOA), 12 benchmark functions are selected and compared with particle swarm optimization (PSO), gray wolf optimization (GWO) and standard whale optimization algorithm (WOA). The 12 benchmark functions are shown in Table 1.

In this work, the values of population size and $M_{a x}$ iter of all algorithms are 30 and 500 respectively. The initial parameter settings of each algorithm used in the experiment are shown in Table 2 .

To test the optimization performance of MSWOA, Table 3 shows the test results of PSO, GWO, WOA and MSWOA after 30 independent experiments, which are compared from the average value (AVG) and standard deviation (STD) of the optimal target value.

It can be seen from Table 3 that among the 12 benchmark functions, the MSWOA algorithm proposed in this paper has the best results in 10 test functions(F1-F10), and only the results of function F11 and F12 are slightly worse than other algorithms. Taking the average value and standard deviation of the optimal solution as the evaluation criteria, it can be seen that the solution accuracy of the MSWOA is significantly better than the other three intelligent algorithms and has strong robustness. In order to more intuitively reflect the convergence speed and the ability to jump out of local optimal value of MSWOA, the convergence curves of each algorithm are shown in Fig.5.

It can be seen from the convergence curve in Fig.5 that MSWOA has the fastest convergence speed in the optimization process of 12 benchmark functions compared with PSO, GWO and standard WOA, which can effectively save optimization time. The convergence curve of the MSWOA algorithm has sudden changes in the decline range, especially when optimizing the five functions of F1, F2, F3, F4 and F5, there are multiple inflection points, which proves that its ability to jump out of the local optimum has been effectively enhanced. Therefore, compared with other intelligent algorithms, MSWOA has a stronger ability to jump out of the local optimum.

\subsection{Selection of Sliding Window and Hidden Layer Node}

For the determination of the size of the sliding window and the number of nodes in the hidden layer, based on the basic criteria proposed in [45], this paper uses comparative experiment to compare the PSNR results after denoising through BP neural network for lena noised images with a Gaussian noise intensity (variance) of 0.05 when the size of the sliding window is 3,5 and 7 and the number of nodes in the hidden layer is 13, 15 and 17 respectively. The experimental results are shown in Table 4. Based on the comparison results, in the next experiment, the sliding window size is $5 * 5$, and the number of hidden layer nodes is 15 .

\subsection{Image Denoising Experiment 1:Benchmark Images}

In this experiment, the Lena image with the size of $512 * 512$ is used as the training sample of neural network. For the benchmark image, the imnoise() function in Matlab is used to add Gaussian noise with a mean value of 0 and different variances. The image after adding the Gauss noise is shown in Fig.6. For the noised images with different noise intensity, Wiener filtering, Neighborhood average filtering, Median 
filtering, BP neural network, WOA-BP and MSWOA-BP are used to denoise them respectively. The PSNR results after denoising are shown in Table 5.

It can be seen from Table 5 that as the noise intensity increases, the denoising effect of every denoising algorithm gradually weakens. Under the same noise intensity, the peak signal-to-noise ratio of MSWOA-BP is the highest, and the denoising effect is the best. In the case of Gaussian noise with a variance of 0.03 , the maximum PSNR of MSWOA-BP for Lena noised images is 28.32.

In order to further verify the denoising effect(generalization ability) of MSWOABP, 4 benchmark images(Man,Boat,Butterfly,House) are selected for testing. The size of Man image and Boat image is $512 * 512$, the size of Butterfly image and House image is $256 * 256$. The Gaussian noise added into the images has a variance of 0.03 . The trained network is used for denoising, and the results are shown in Fig. 7 and Table 6.

It can be seen from Table 6 that the PSNR of MSWOA-BP are all less than 28.32, which shows that its generalization denoising effect is limited by the denoising ability of the training network, but the two values are close (the maximum difference is 3.61 ), indicating that the trained network has good generalization and denoising ability. Compared with traditional Median filtering, Neighborhood average filtering and Wiener filtering, MSWOA-BP has a higher PSNR, and compared with BP and WOA-BP, MSWOA-BP also has certain advantages in PSNR.

\subsection{Image Denoising Experiment 2:Traffic Images}

In this section, 6 traffic images from UAS (UESTC All-Day Scenery) [46] data set are selected and grayed for denoising experiment, as shown in Fig.8,named TI-1,TI2,TI-3,TI-4,TI-5,TI-6 respectively. The size of each image is $640 * 360$. Fig.8(a) is used for training the neural networks, and Fig.8(b)-(f) are used for verifying the denoising effect. The intensity of Gaussian noise is 0.01 . The experimental results are shown in Table 7 and Fig.9.

Table 7 shows PSNR with different denoising algorithms for traffic images.It can be seen that for each traffic image, the denoising effect of MAWOA-BP is the best, which is obviously better than Median filtering, Neighborhood average filtering and Wiener filtering. Fig.9 shows denoising effect of different denoising algorithms for Fig.8(d). From the perspective of visual effect, each denoising algorithm has a certain denoising effect. The denoising result of Wiener filtering makes the image boundary more fuzzy, while the MSWOA-BP denoising result image is more visible.

\section{Conclusion}

Traffic image denoising is an useful technique for intelligent transportation system in smart city construction. This paper proposes an image denoising method based on BP neural network optimized by improved whale optimization algorithm to denoise the traffic image and improve the image quality and visibility. The improved whale optimization algorithm is used to optimize the initial weights and thresholds of the BP neural network to overcome the dependence of the initial weights and thresholds in the construction process of the BP neural network, and shortens the training time of the neural network. The improved whale optimization algorithm's ability to jump out of the local optimum has been effectively enhanced. And compared with other 
intelligent optimization algorithms, the convergence speed of it is also improved. The experimental results of image denoising show that compared with the traditional Median filtering, Neighborhood average filtering, Wiener filtering algorithm and other denoising algorithms, MSWOA-BP has a significant improvement in the peak signal-to-noise ratio (PSNR). Since the generalization and denoising ability of MSWOA-BP is limited by the training effect of the network, further improving the training effect of MSWOA-BP neural network will be taken as the next step of research.

\begin{abstract}
Abbreviations
IoT:Internet of Things;ITS:Intelligent transportation system;WOA:Whale optimization algorithm;BP:Back propagation;PSO:Particle swarm optimization;GSA:Gravitational search algorithm;UAS:UESTC All-Day Scenery;PDE:Partial differential equation; CWOA:Chaotic whale optimization algorithm;MSWOA:Improved whale optimization algorithm based on mixed strategy; GWO:Gray wolf optimization;MSE:Mean square error;MF:Median filtering;NAF:Neighborhood average filtering;WF:Wiener filtering;PSNR:Peak signal-to-noise ratio;AVG:Average value;STD:Standard deviation.
\end{abstract}

Availability of Data and Materials

The datasets used and analyzed during the current study are available from the corresponding author on reasonable request.

Competing Interest

The authors declare that they have no conflict of interest.

Funding

Authors receive research support from National Natural Science Foundation of China (Grant No.61772180), Technological innovation project of Hubei Province 2019 (2019 AAA047), Green Industry Science and Technology Leadership Program of Hubei University of Technology (No.CPYF2018005) and Hubei province graduate education innovation plan.

Authors' Contribution

CW conceived the idea of the study, and participated in the design and coordination and helped to revised the manuscript. RW and HY contributed to refining the ideas. ML and SW performed the research, collected the data, analysed the results and drafted the manuscript. All authors read and approved the final manuscript.

Author details

${ }^{1}$ School of Computer Science, Hubei University of Technology, Wuhan, China. ${ }^{2}$ Wuhan Fiberhome Technical Services Co.,Ltd.. ${ }^{3}$ Fiberhome Telecommunication Technologies Co.,Ltd.. ${ }^{4}$ Department of Information Statistics, Hubei Cancer Hospital,Tongji Medical College, Huazhong University of Science and Technology, Wuhan, China.

\title{
References
}

1. Gonzalez, R.A., Ferro, R.E., Liberona, D.: Government and governance in intelligent cities, smart transportation study case in bogotá colombia. Ain Shams Engineering Journal 11(1), 25-34 (2020). doi:10.1016/j.asej.2019.05.002

2. Molnar, A.: Smart cities education: An insight into existing drawbacks. Telematics and Informatics (2020). doi:10.1016/j.tele.2020.101509

3. Saborido, R., Alba, E.: Software systems from smart city vendors. Cities 101, 102690 (2020). doi:10.1016/j.cities.2020.102690

4. Rani, R., Kashyap, V., Khurana, M.: Role of iot-cloud ecosystem in smart cities : Review and challenges. Materials Today: Proceedings (2020). doi:10.1016/j.matpr.2020.10.054

5. Yan, J., Liu, J., Tseng, F.M.: An evaluation system based on the self-organizing system framework of smart cities: A case study of smart transportation systems in china. Technological Forecasting and Social Change $\mathbf{1 5 3}$ (2020)

6. Saharan, S., Bawa, S., Kumar, N.: Dynamic pricing techniques for intelligent transportation system in smart cities: A systematic review. Computer Communications 150 (2019). doi:10.1016/j.comcom.2019.12.003

7. Karami, Z., Kashef, R.: Smart transportation planning: Data, models, and algorithms. Transportation Engineering 2, 100013 (2020). doi:10.1016/j.treng.2020.100013

8. Agachai, S., Wai, H.H.: Smarter and more connected: Future intelligent transportation system. IATSS Research 42, 67-71 (2018). doi:10.1016/j.iatssr.2018.05.005

9. Siuhi, S., Mwakalonge, J.: Opportunities and challenges of smart mobile applications in transportation. Journal of Traffic and Transportation Engineering (06), 96-106 (2016). doi:10.1016/j.jtte.2016.11.001

10. WANG, H., MENKE, N., JIN, T.: The application of bat neural network algorithm in image denoising. Microelectronics and Computer 35, 121-124 (2018). doi:10.19304/j.cnki.issn1000-7180.2018.09.026

11. WANG, H.: Researching image denoising model based pso-trainlm bp. Mathematics in Practice and Theory 44(21), 137-142 (2014)

12. YUAN, M., CHEN, G.: Study on improved algorithm of median filtering based on grey correlation. Geomatics Spatial Information Technology 43(5), 124-127130 (2020) 
13. Zhang, W., Liang, C., Gao, X.: Design of median filtering algorithm with multistage threshold. Computer Era 5, 9-12 (2020). doi:10.16644/j.cnki.cn33-1094/tp.2020.05.003

14. SUN, T., CUI, S.: Denoising method of super gaussian signal based on kurtosis ica and eigen image filtering Journal of Hebei Normal University(Natural Science Edition) 44, 209-214 (2020). doi:10.13763/j.cnki.jhebnu.nse.2020.03.004

15. XU, R., WANG, Z., ZONG, T.: Edge enhancement of medical image based on improved gaussian filter. Information Technology 44(4), 75-78 (2020). doi:10.13274/j.cnki.hdzj.2020.04.016

16. Xue, H., Cui, H.: Research on image restoration algorithms based on bp neural network. Journal of Visual Communication and Image Representation 59, 204-209 (2019). doi:10.1016/j.jvcir.2019.01.014

17. Zuo, C.: Research on image nonlocal mean denoising method. Phd thesis, National University of Defense Science and technology (2016)

18. SU, J., YANG, W.: Image segmentation algorithm based on bp neural network. Industrial Control Computer 28(12), 2932- (2015)

19. Duan, F., Xiong, X., Han, X.: A new method for image segmentation based on bp neural network and gravitational search algorithm enhanced by cat chaotic mapping. Applied Intelligence: The International Journal of Artificial Intelligence, Neural Networks, and Complex Problem-Solving Technologies 43(4), 855-873 (2015). doi:10.1007/s10489-015-0679-5

20. WU, Y., GAO, R., YANG, J.: Prediction of coal and gas outburst: A method based on the bp neural network optimized by gasa. Process Safety and Environmental Protection 133, 64-72 (2020). doi:10.1016/j.psep.2019.10.002

21. Mirjalili, S., Lewis, A.: The whale optimization algorithm. Advances in engineering software 95, 51-67 (2016). doi:10.1016/j.advengsoft.2016.01.008

22. LEI, X., OUYANG, H., XIAO, L., FAN, C.: Research on image segmentation based on equivalent 3-d entropy and whale optimization algorithm. Computer Engineering 45(4), 217-222 (2019). doi:10.19678/j.issn.1000-3428.0049933

23. Aziz, A.E., Mohamed, Ewees, A., A., Hassanien, Ella, A.: Whale optimization algorithm and moth-flame optimization for multilevel thresholding image segmentation. Expert Systems with Application 83(Oct.), 242-256 (2017). doi:10.1016/j.eswa.2017.04.023

24. Yuan, Z., Xie, X., Hu, J., Yao, D.: An efficient method for traffic image denoising. Procedia - Social and Behavioral Sciences 138, 439-445 (2014). doi:10.1016/j.sbspro.2014.07.222

25. Shijie, J., Yanbing, L.: A traffic image denoising algorithm based on sparse representation. Journal of Dalian Jiaotong University 34(005), 107-111 (2013). doi:10.3969/j.issn.1673-9590.2013.05.025

26. Dongxu, C., Yan, Y.: Study on the license plate image denoising based on adaptive coupling pde model. Computer Measurement and Control 022(008), 2592-2594 (2014)

27. JING, L., SHAO, D., XIANG, Y., MA, L., YANG, Z., ZHU, X.: Application of bp neural network in medical ultrasound image denoising. Data Communications 5, 18-21 (2019)

28. YAN, L., WANG, L.: Copula estimation of distribution algorithm based on centroid and its application in image denoising. Computer Engineering 42(2), 195-199205 (2016)

29. WANG, H., LI, P., WANG, B., ZHAI, S., CAI, N.: Image deblurring restoration of bp neural network based on grey wolf algorithm. Chinese Journal of Liquid Crystals and Displays 34(10), 992-999 (2019)

30. WANG, H.: Research on image restoration method based on improved gray wolf algorithm-bp neural network. master thesis, Ningxia University (April 2019). doi:10.27257/d.cnki.gnxhc.2019.000344

31. He Qing, X.- Wei Kangyuan: Improved whale optimization algorithm based on hybrid strategy. Application Research of Computer 36, 3647-36513665 (2019). doi:10.19734/j.issn.1001-3695.2018.07.0382

32. Arora, G.K.: Chaotic whale optimization algorithm. Journal of Computational Design and Engineering $\mathbf{5}$, 275-284 (2018). doi:10.1016/j.jcde.2017.12.006

33. Mirjalili, M.M.M.: Hybrid whale optimization algorithm with simulated annealing for feature selection. Neurocomputing 260 (2017). doi:10.1016/j.neucom.2017.04.053

34. Oliva, M.A.E.: Parameter estimation of solar cells diode models by an improved opposition-based whale optimization algorithm. Energy Conversion and Management 171 (2018). doi:10.1016/j.enconman.2018.05.062

35. Zhang Yong, C.F.: An improved whale optimization algorithm. Computer Engineering 44, 208-213219 (2018)

36. Long Wen, J.J.e.a. Cai Shaohong: Improved whale optimization algorithm for solving large-scale optimization problems. System Engineering-Theory and Practice 37, 2983-2994 (2017)

37. Liu Liang, H.Q.: Improved whale optimization algorithm for solving function optimization problems. Application Research of Computers 37, 1004-1009 (2020). doi:10.19734/j.issn.1001-3695.2018.11.0726

38. Wu Zequan, M.Y.: Improved whale optimization algorithm. Application Research of Computers 37 (2020). doi:10.19734/j.issn.1001-3695.2019.09.0536

39. Zhang Shuiping, G.D.: Dynamic search and cooperative learning for whale optimization algorithm. Application Research of Computers 37 (2019). doi:10.19734/j.issn.1001-3695.2019.05.0119

40. CHEN, Z.: Prediction of soil parameters based on back propagation neural network optimized by genetic algorithm whale algorithm. Journal of Zhejiang Agricultural Sciences 60(1), 125-128140 (2019) doi:10.16178/j.issn.0528-9017.20190140

41. Hao Xiaohong, Z.Q.e.a. Song Jixiang: Improved whale optimization algorithm based on hybrid strategy. Application Research of Computers 37 (2020). doi:10.19734/j.issn.1001-3695.2019.09.0528

42. LI, D., LI, J., ZHANG, Y., ZENG, Z.: Gesture recognition of data glove based on pso-improved bp neural network. Electric Machines and Control 18(8), 87-93 (2014). doi:10.15938/j.emc.2014.08.016

43. WU, X., YAO, N., XU, J.: Substation transformer crack image recognition based on improved neural network algorithm. Modern Electronics Technique 40(13), 66-69 (2017). doi:10.16652/j.issn.1004-373x.2017.13.017

44. BI, S.: Sonar image segmentation based on bp neural network optimized by genetic algorithm. master thesis, Inner Mongolia University (2018)

45. Xia Kewen, S.J. Li Changbiao: An optimization algorithm on the number of hidden layer nodes in feed-forward neural network. Computer Science 32(10), 143-145 (2005) 
46. Zhang, Y., Chen, H., He, Y., Ye, M., Cai, X., Zhang, D.: Road segmentation for all-day outdoor robot navigation. Neurocomputing 314, 316-325 (2018)

Figures

Figure 1 Bubble-net feeding behavior of humpback whales

Figure 2 BP neural network structure

Figure 3 Change of the improved convergence factor $a$

Figure 4 Principle of image denoising using BP neural network

Figure 5 Convergence curve of MSWOA and other algorithms

Figure 6 Lena image with different Gaussian noise:(a) without noise; (b) variance of 0.03 ; (c) variance of 0.04 ; (d) variance of 0.06 ; (e) variance of 0.08 .

Figure 7 Denoising effect of different denoising algorithms for benchmark images

Figure 8 Original traffic images:(a) TI-1;(b) TI-2;(c) TI-3;(d) TI-4;(e) TI-5;(f) TI-6

Figure 9 Denoising effect of different denoising algorithms for traffic images:(a) original image,(b) noised image,(c) Median flitering result,(d) Neighborhood average filtering result,(e) Wiener filtering result,(f) BP result,(g) WOA-BP result,(h) MSWOA-BP result

Tables 
Table 112 benchmark functions

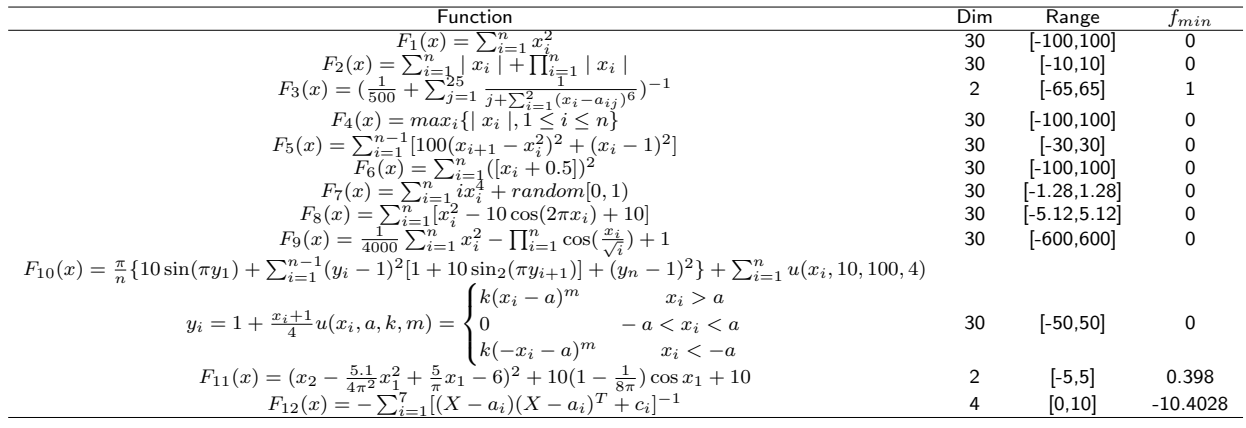

Table 2 Parameter setting

\begin{tabular}{ccc}
\hline Algorithm & Parameter & Value \\
\hline \multirow{2}{*}{ PSO } & $c_{1}$ & 2 \\
& $c_{2}$ & 2 \\
GWO & $\omega$ & 0.9 linearly decreases to 0.2 \\
WOA & $a$ & 2 linearly decreases to 0 \\
MSWOA & $a$ & 2 linearly decreases to 0 \\
& $a$ & 2 nonlinearly decreases to 0 \\
& $\omega$ & 0 nonlinearly increases to 1 \\
\hline
\end{tabular}

Table 3 Performance comparison of four algorithms

\begin{tabular}{ccccccccc}
\hline \multirow{2}{*}{ F } & \multicolumn{2}{c}{ PSO } & \multicolumn{2}{c}{ GWO } & \multicolumn{2}{c}{ WOA } & \multicolumn{2}{c}{ MSWOA } \\
& avg & std & avg & std & avg & std & avg & std \\
\hline F1 & 0.000201 & 0.000358 & $9.8969 \mathrm{e}-28$ & $1.7798 \mathrm{e}-27$ & $1.3287 \mathrm{e}-71$ & $4.6923 \mathrm{e}-71$ & $2.987 \mathrm{e}-235$ & 0.000000 \\
F2 & 0.027834 & 0.022819 & $9.4228 \mathrm{e}-17$ & $4.0359 \mathrm{e}-17$ & $6.5191 \mathrm{e}-52$ & $1.3916 \mathrm{e}-51$ & $7.4087 \mathrm{e}-122$ & $2.7436 \mathrm{e}-121$ \\
F3 & 3.2627 & 2.829 & 3.254 & 3.5172 & 2.537 & 2.8982 & 1.1964 & 0.60541 \\
F4 & 1.0994 & 0.20903 & $6.1646 \mathrm{e}-07$ & $5.0583 \mathrm{e}-07$ & 43.683 & 30.5652 & $2.9716 \mathrm{e}-120$ & $1.5375 \mathrm{e}-119$ \\
F5 & 82.3794 & 94.0824 & 27.9435 & 0.85738 & 27.996 & 0.53038 & 27.8959 & 0.1526 \\
F6 & $9.3017 \mathrm{e}-05$ & 0.000108 & 0.80641 & 0.41776 & 0.39688 & 0.23078 & 0.20277 & 0.065166 \\
F7 & 0.17078 & 0.057366 & 0.002217 & 0.001334 & 0.003305 & 0.004761 & $8.907 \mathrm{e}-05$ & $6.9351 \mathrm{e}-05$ \\
F8 & 56.481 & 15.5315 & 2.8209 & 3.4839 & 0.000000 & 0.000000 & 0.000000 & 0.000000 \\
F9 & 0.009372 & 0.009238 & 0.002068 & 0.005518 & 0.011227 & 0.042789 & 0.000000 & 0.000000 \\
F10 & 0.010368 & 0.041732 & 0.041238 & 0.016416 & 0.032643 & 0.044017 & 0.007025 & 0.002786 \\
F11 & 0.39789 & 0.000000 & 0.39789 & $4.6716 \mathrm{e}-06$ & 0.39789 & $8.4987 \mathrm{e}-06$ & 0.39878 & 0.003067 \\
F12 & -8.5514 & 3.1639 & -10.4012 & 0.000910 & -7.8285 & 2.963 & -8.0695 & 2.3667 \\
\hline
\end{tabular}

Table 4 Performance of BP neural network with different number of hidden layer nodes

\begin{tabular}{|c|c|c|}
\hline size of the sliding window & number of hidden layer node & PSNR \\
\hline \multirow{4}{*}{$3 * 3$} & 13 & 18.67 \\
\hline & 15 & 20.67 \\
\hline & 17 & 19.74 \\
\hline & 13 & 22.17 \\
\hline \multirow[t]{3}{*}{$5 * 5$} & 15 & 23.57 \\
\hline & 17 & 21.91 \\
\hline & 13 & 23.44 \\
\hline \multirow[t]{2}{*}{$7^{*} 7$} & 15 & 23.55 \\
\hline & 17 & 22.82 \\
\hline
\end{tabular}

Table 5 PSNR of image denoising

\begin{tabular}{ccccc}
\hline \multirow{2}{*}{ denoising algorithm } & \multicolumn{4}{c}{ noise intensity } \\
& 0.03 & 0.04 & 0.06 & 0.08 \\
\hline MF & 21.20 & 20.00 & 18.91 & 17.86 \\
NAF & 22.11 & 21.12 & 20.25 & 19.41 \\
WF & 22.59 & 21.17 & 20.06 & 19.11 \\
BP & 25.90 & 24.40 & 21.95 & 20.61 \\
WOA-BP & 27.75 & 25.82 & 23.90 & 22.96 \\
MSWOA-BP & 28.32 & 26.77 & 25.11 & 24.18 \\
\hline
\end{tabular}

Table 6 PSNR with different denoising algorithms for benchmark images

\begin{tabular}{ccccccc}
\hline & MF & NAF & WF & BP & WOA-BP & MSWOA-BP \\
\hline Man & 21.09 & 21.88 & 22.48 & 23.59 & 25.67 & 25.97 \\
Boat & 20.82 & 21.46 & 22.27 & 24.98 & 26.04 & 26.18 \\
Butterfly & 19.73 & 20.14 & 22.21 & 23.79 & 24.88 & 25.77 \\
House & 19.31 & 19.84 & 22.57 & 22.88 & 23.80 & 24.71 \\
\hline
\end{tabular}


Table 7 PSNR with different denoising algorithms for traffic images

\begin{tabular}{ccccccc}
\hline & MF & NAF & WF & BP & WOA-BP & MSWOA-BP \\
\hline TI-2 & 24.57 & 25.20 & 26.65 & 28.27 & 28.32 & 28.44 \\
Tl-3 & 25.80 & 26.47 & 26.60 & 26.75 & 27.07 & 27.32 \\
Tl-4 & 25.06 & 25.27 & 26.13 & 26.51 & 26.81 & 28.58 \\
TI-5 & 23.26 & 23.67 & 26.28 & 26.70 & 27.37 & 27.54 \\
TI-6 & 24.40 & 25.00 & 26.47 & 28.23 & 28.55 & 28.68 \\
\hline
\end{tabular}




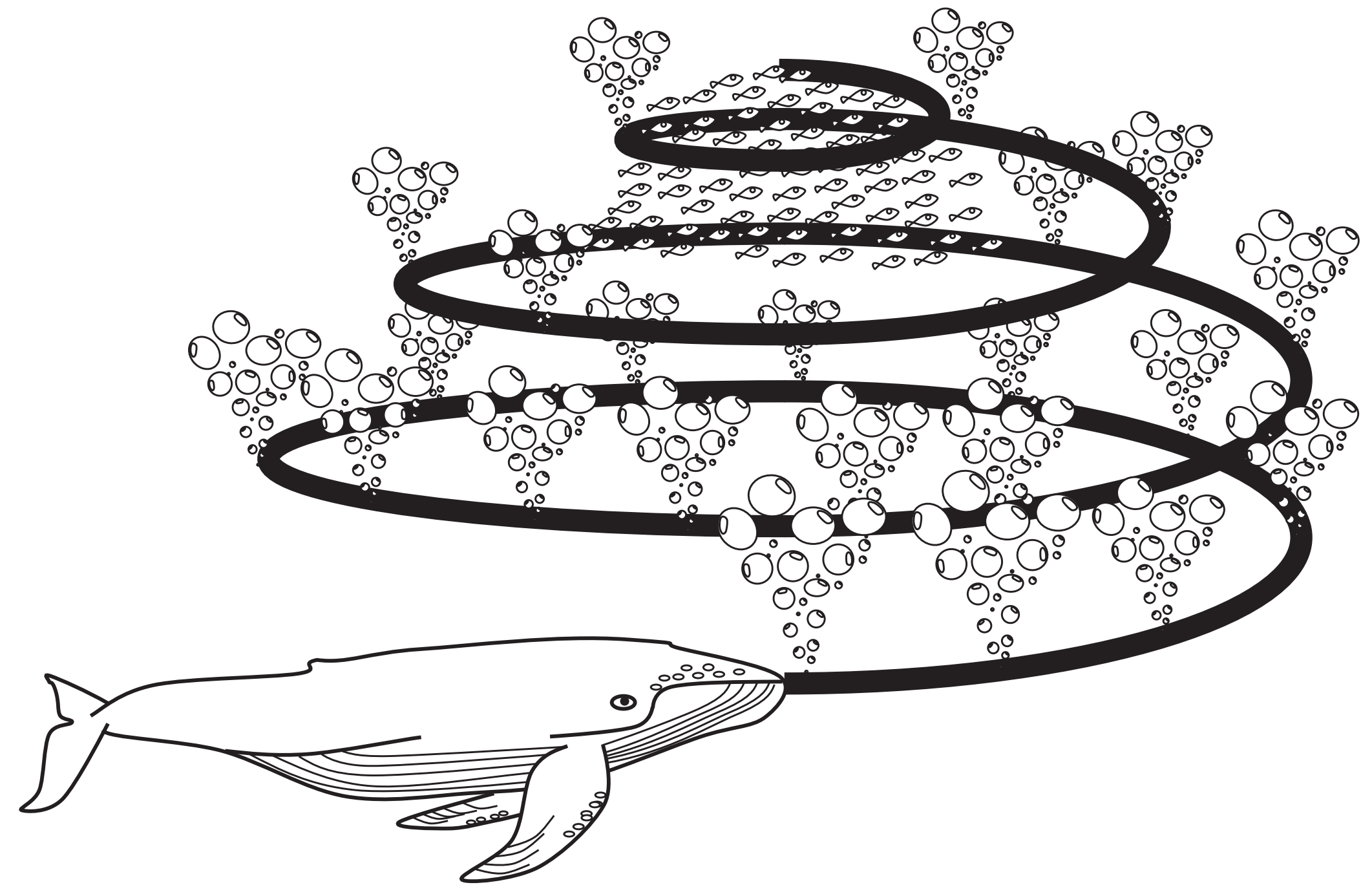


input

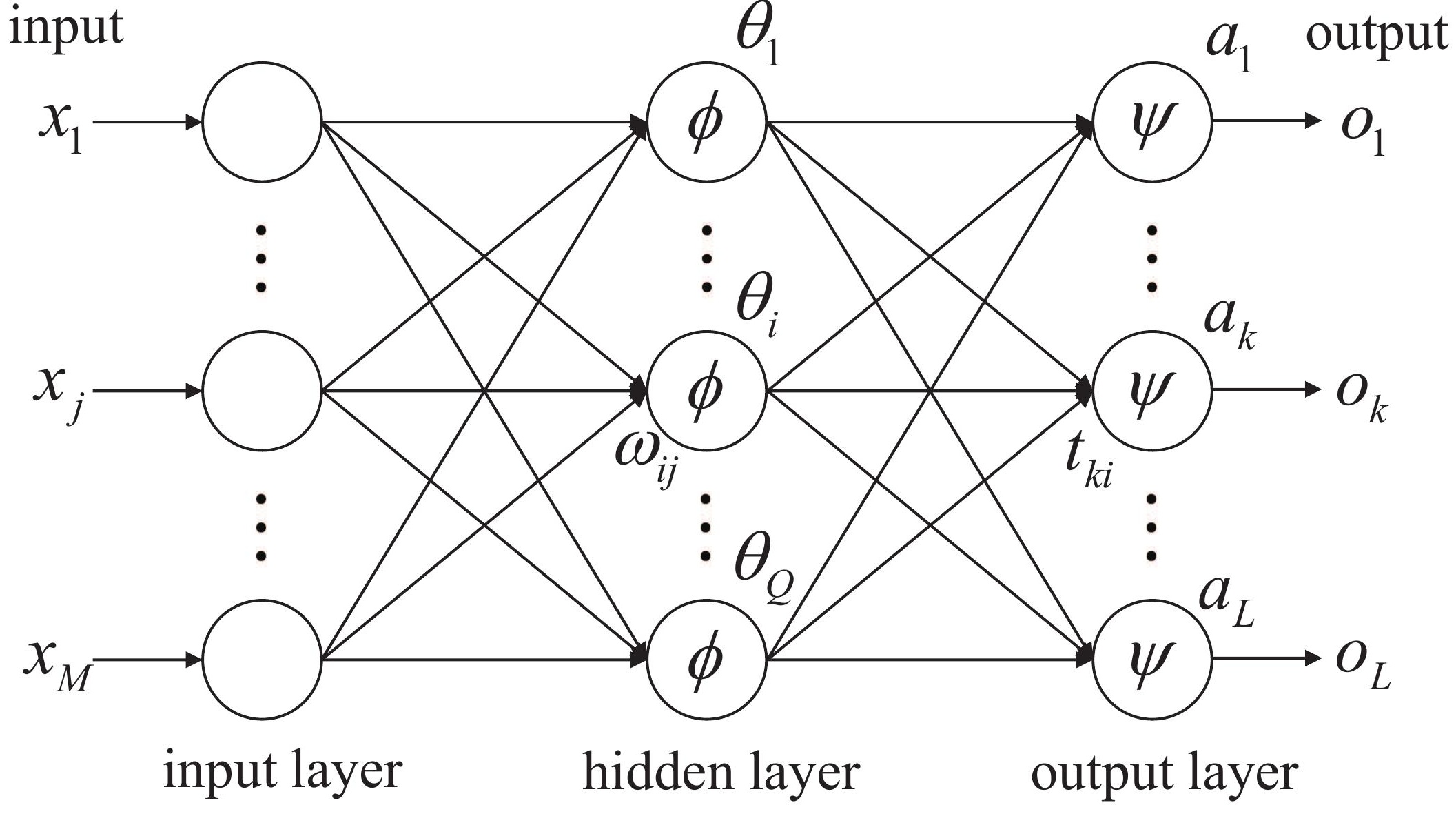




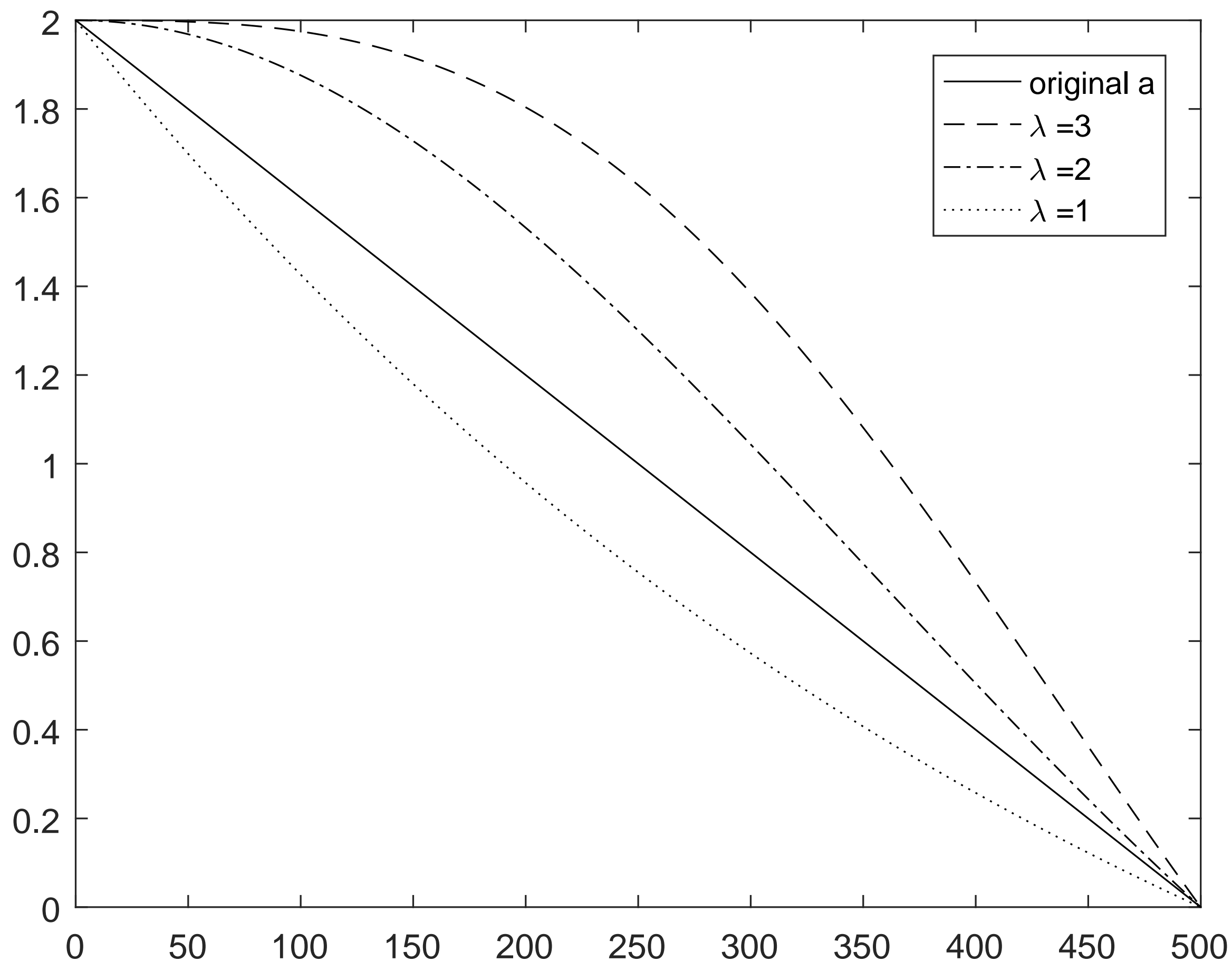




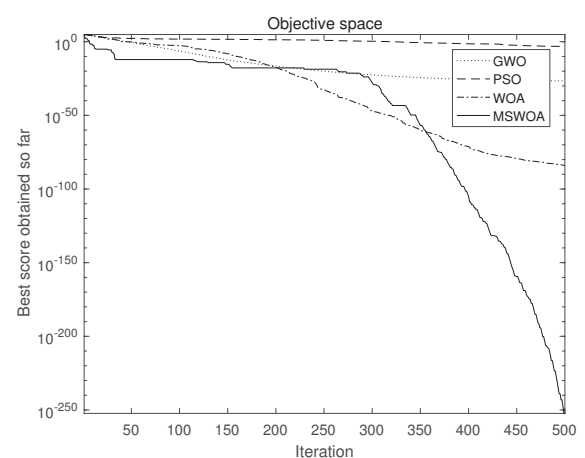

$F_{1}$

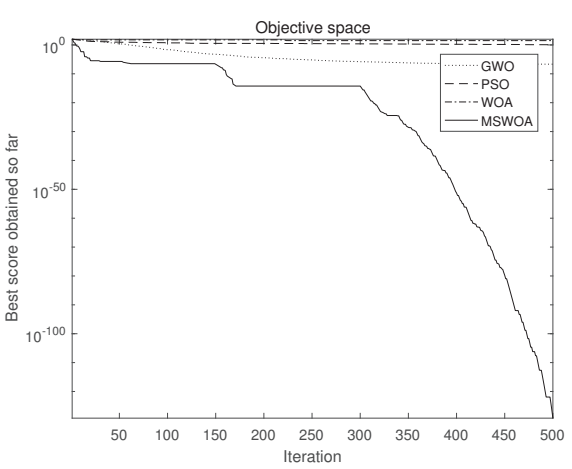

$F_{4}$

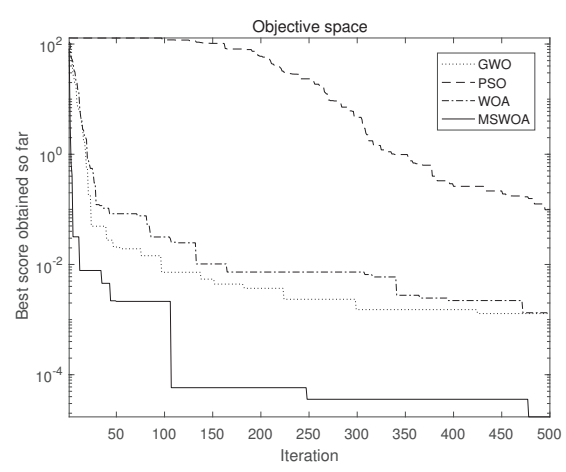

$F_{7}$

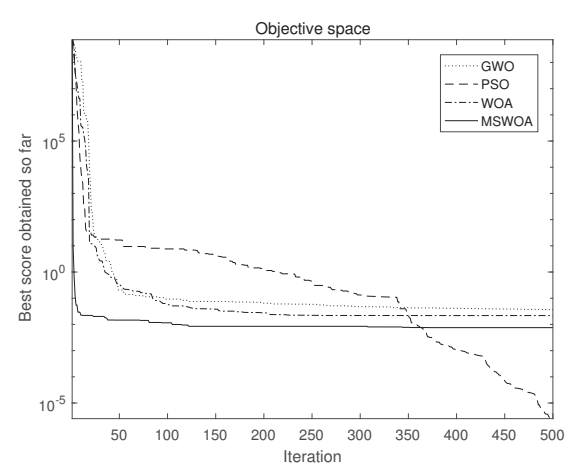

$F_{10}$

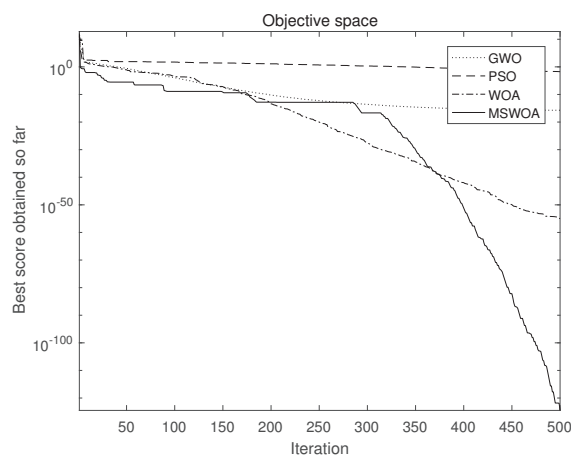

$F_{2}$

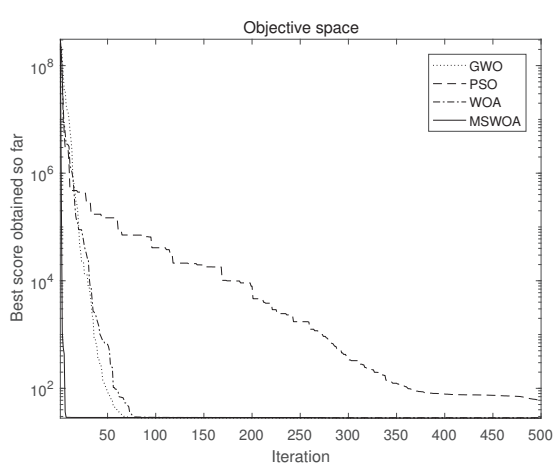

$F_{5}$

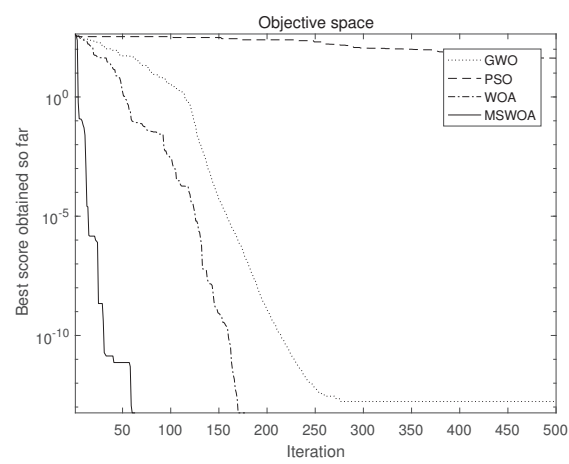

$F_{8}$

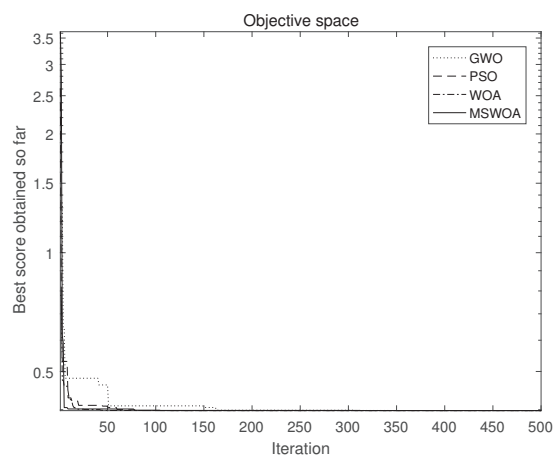

$F_{11}$

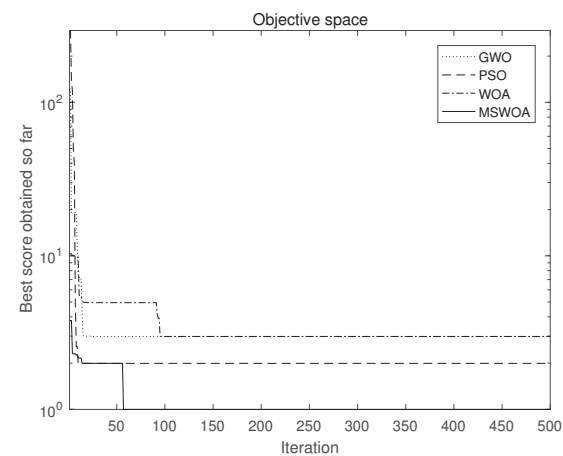

$F_{3}$

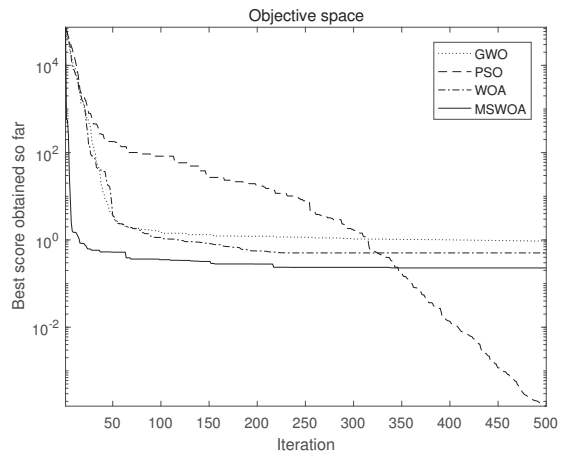

$F_{6}$

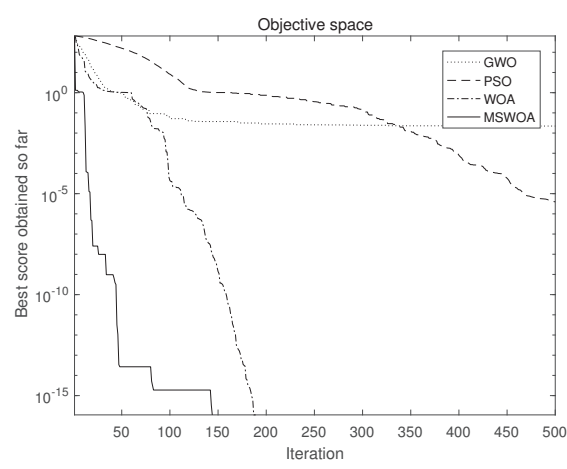

$F_{9}$

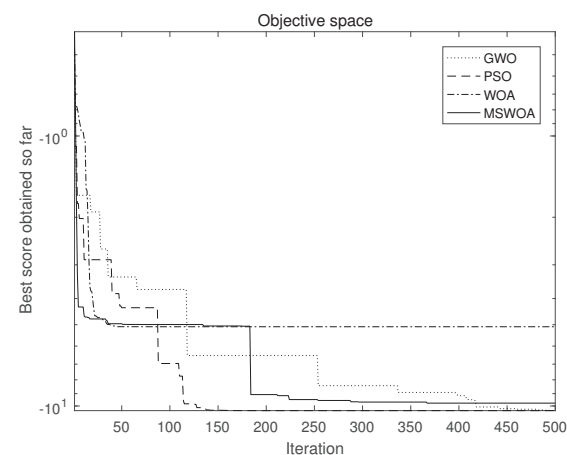

$F_{12}$ 
Figure

Figure

Figure

Figure

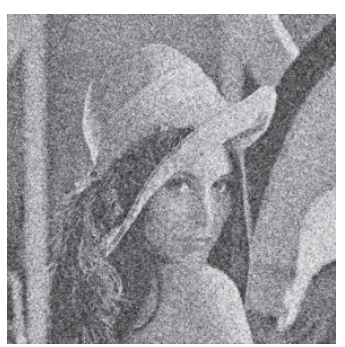

Figure

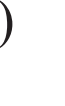

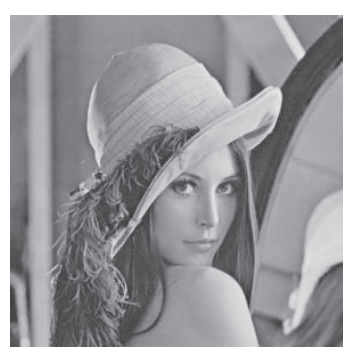

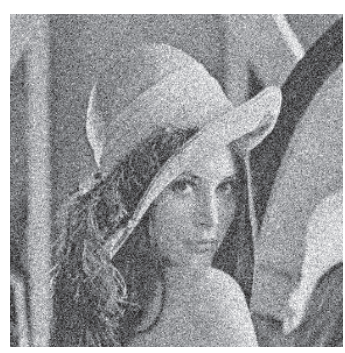

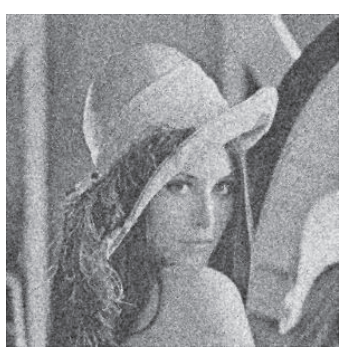

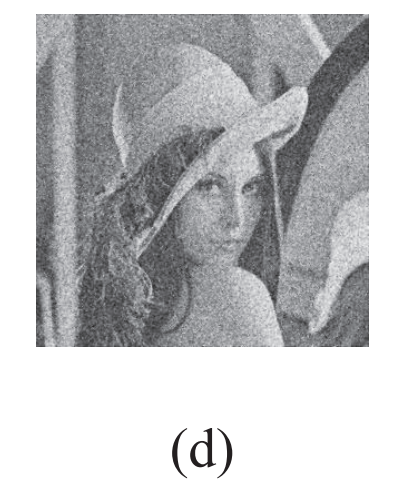
.
(a)
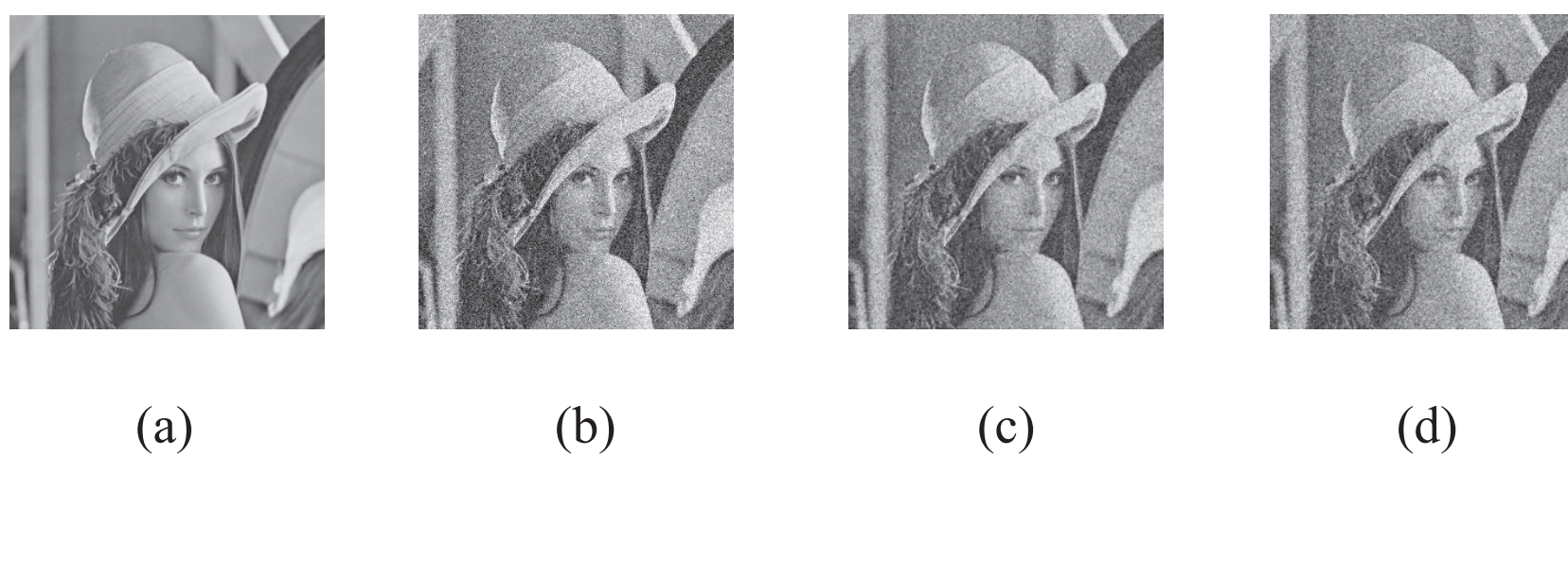
Boat
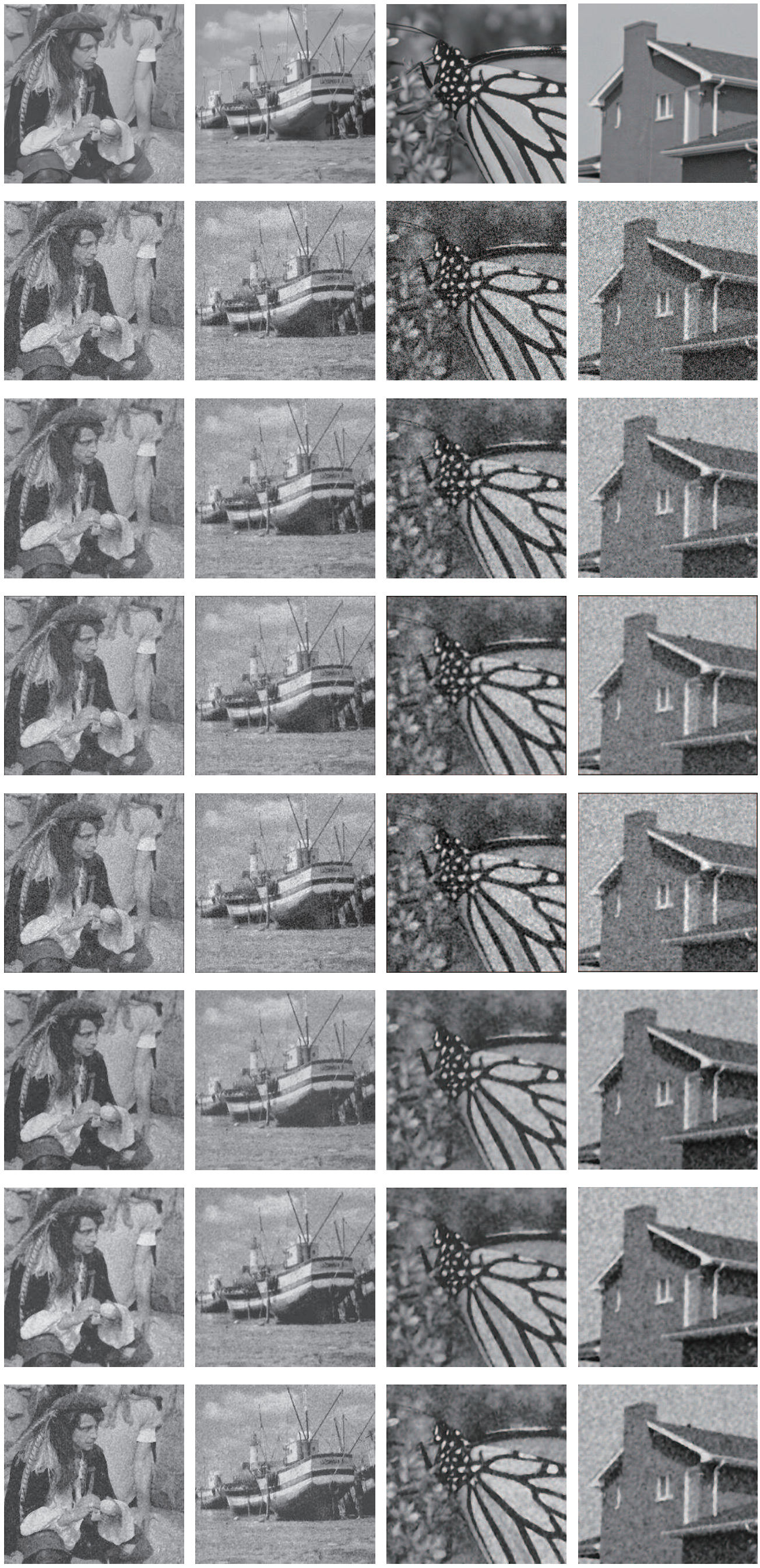
Figures

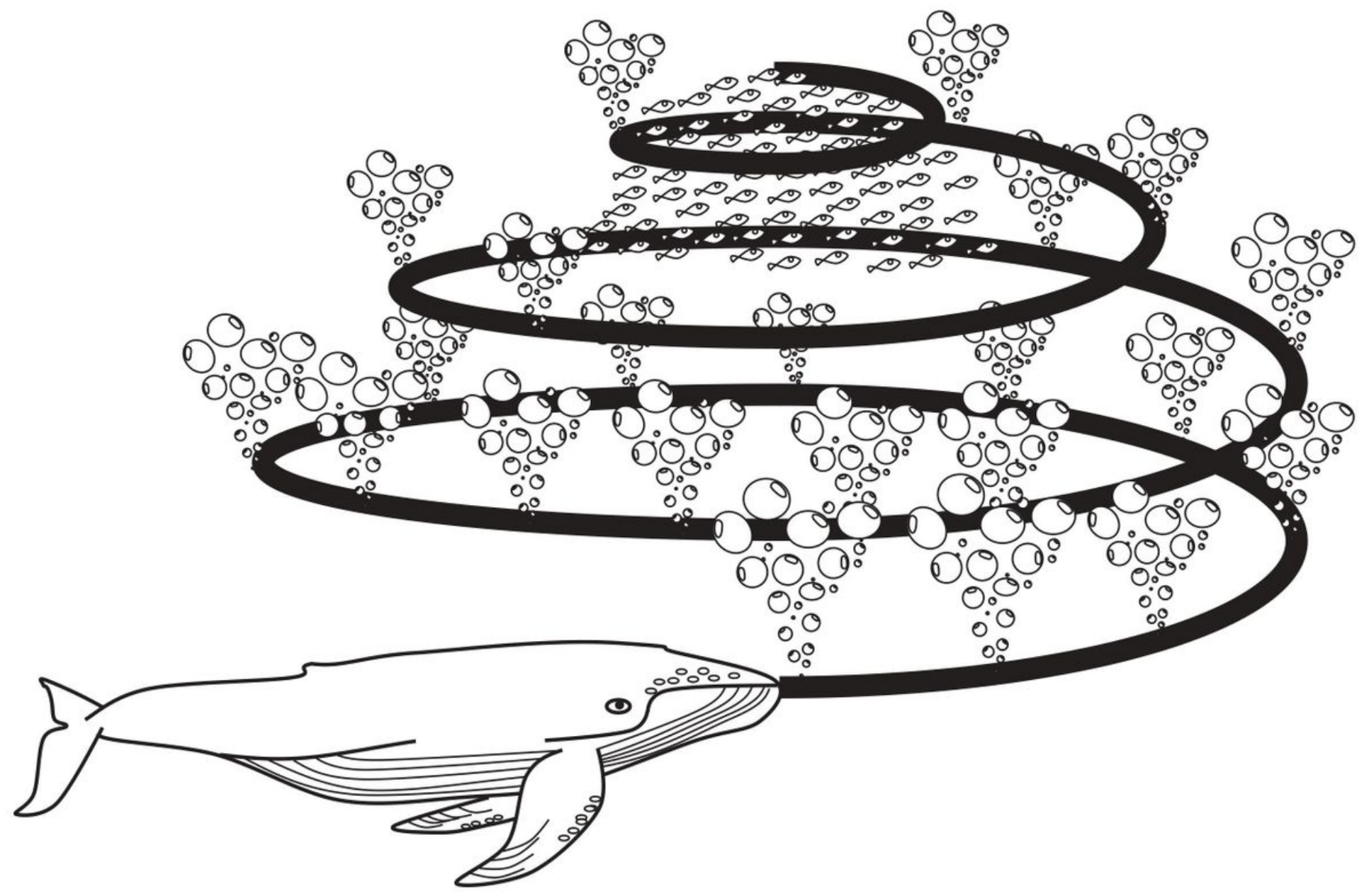

Figure 1

Bubble-net feeding behavior of humpback whales 


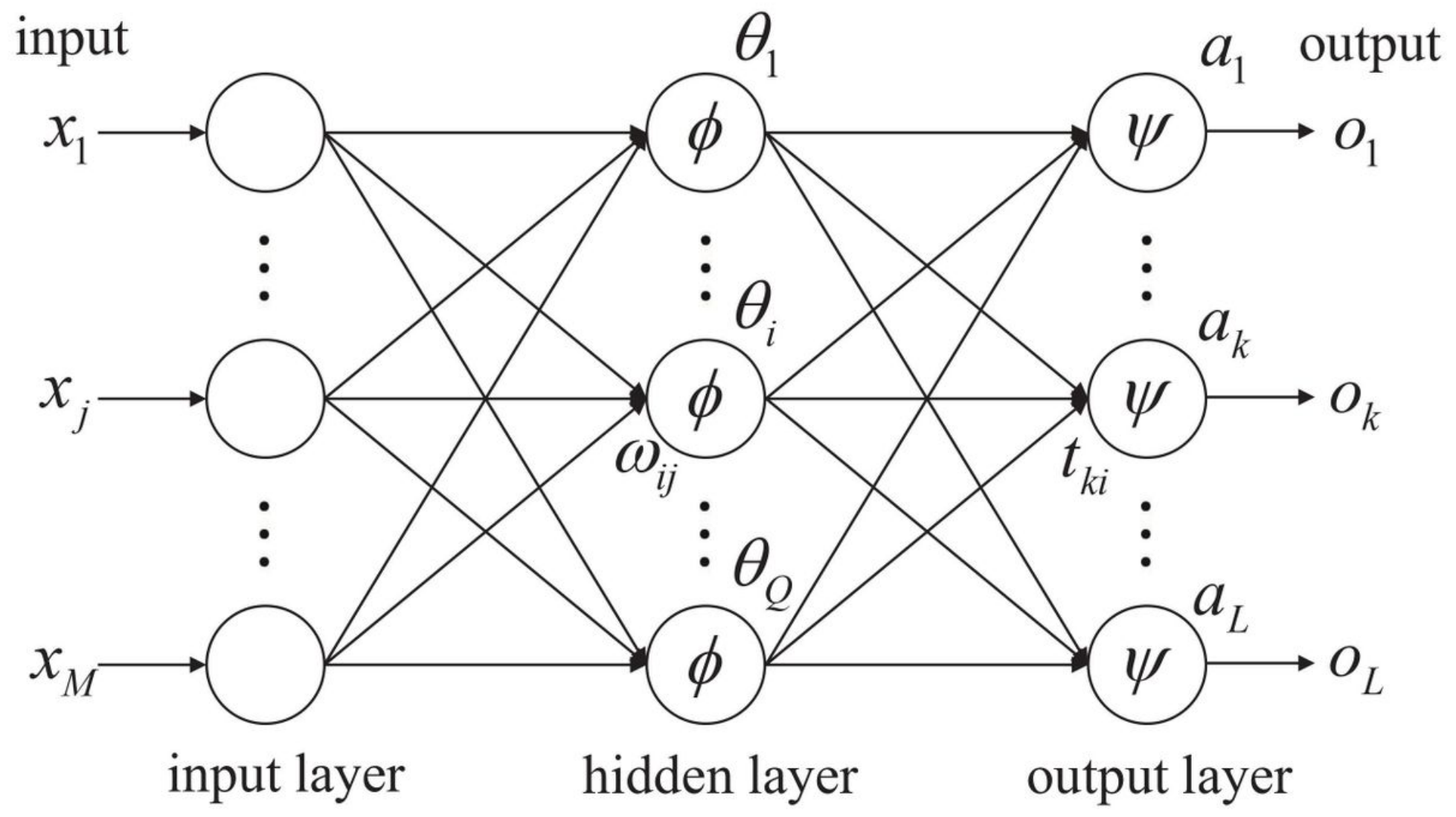

Figure 2

BP neural network structure 


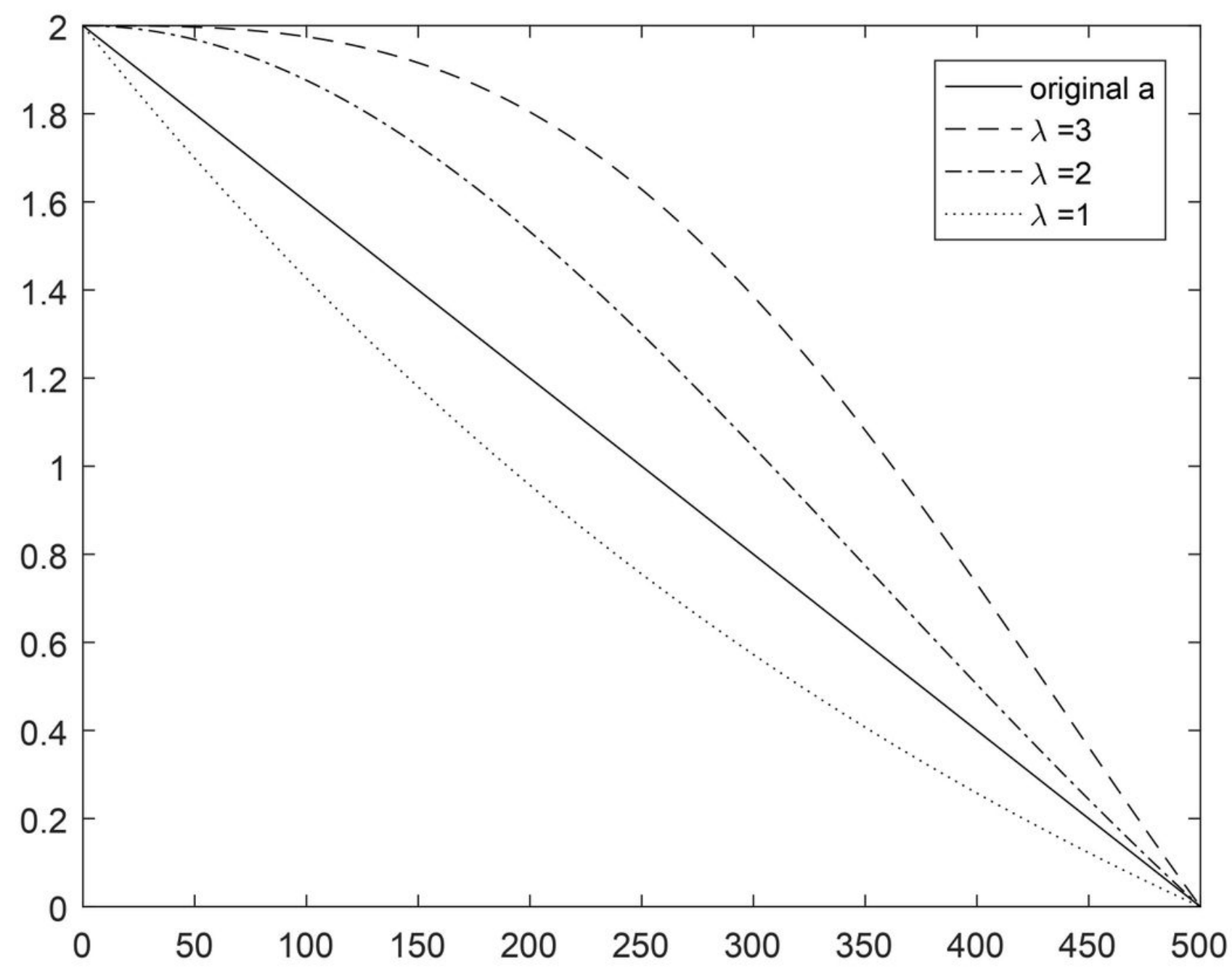

Figure 3

Change of the improved convergence factor a

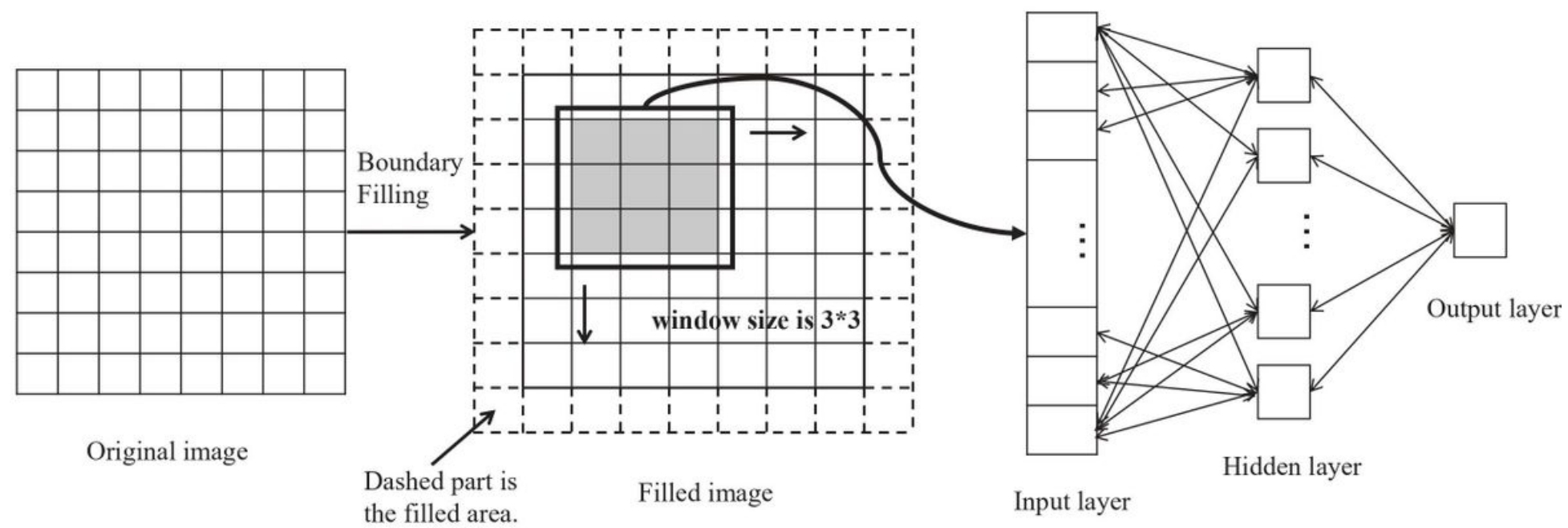


Principle of image denoising using BP neural network
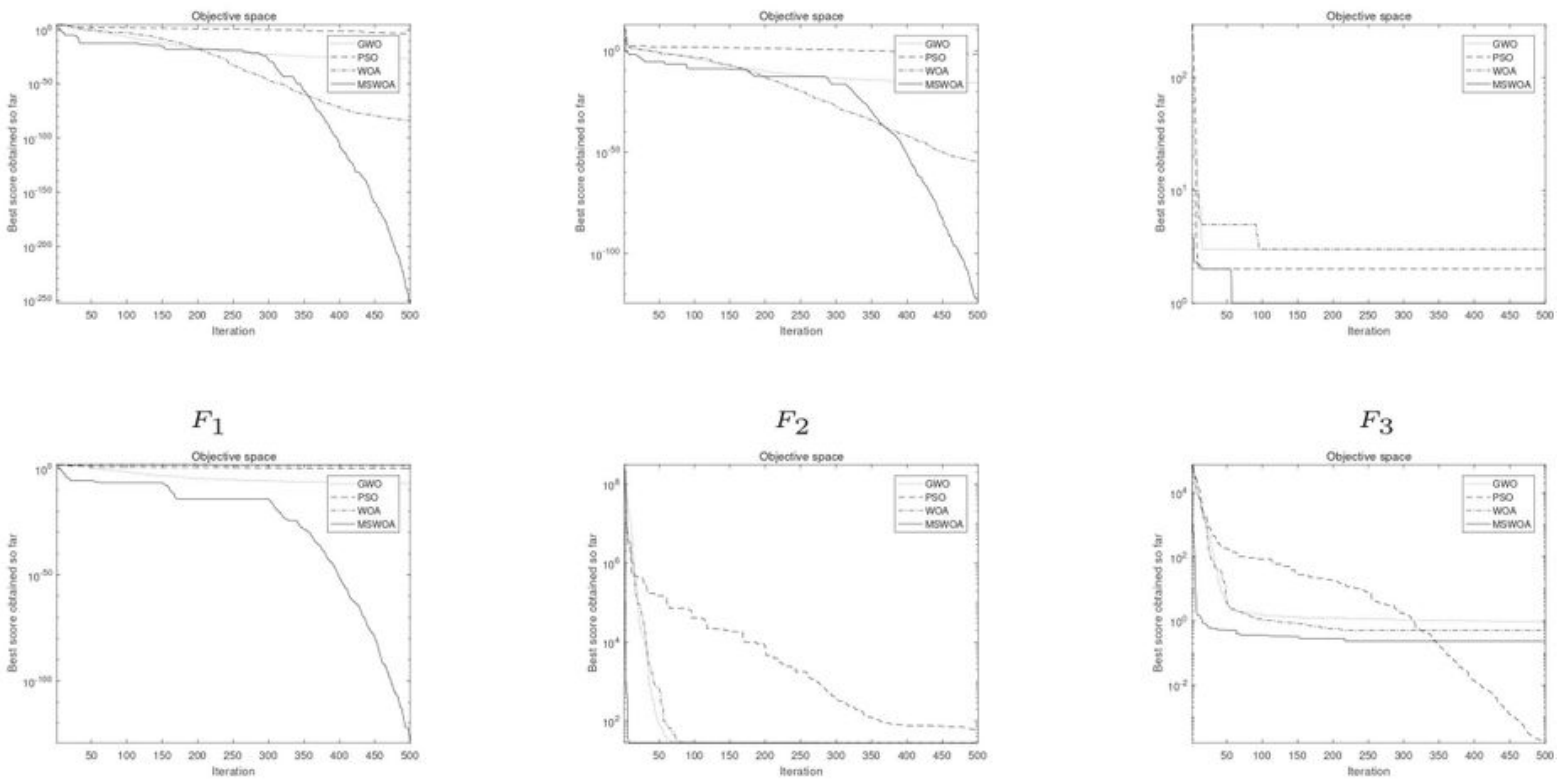

$F_{4}$
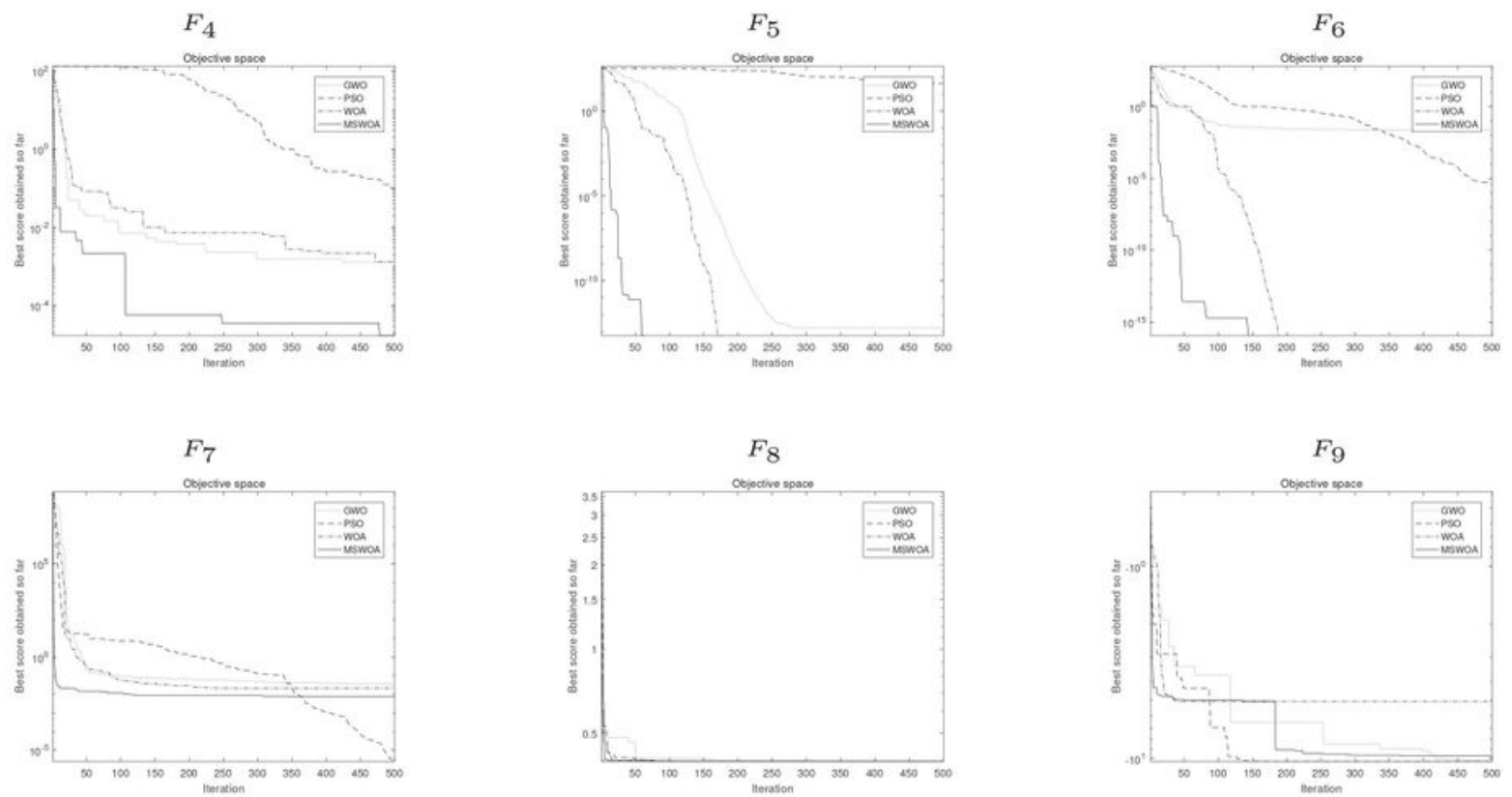

$F_{10}$

$F_{11}$

$F_{12}$

Figure 5

Convergence curve of MSWOA and other algorithms 


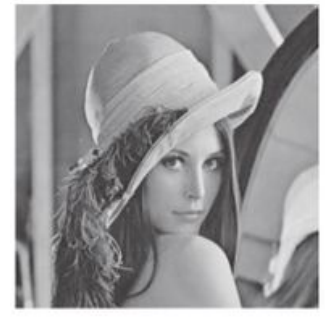

(a)

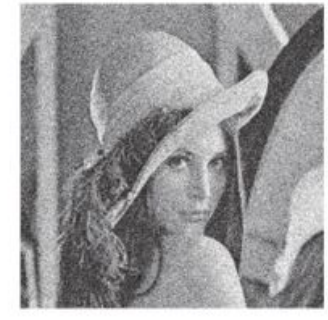

(b)

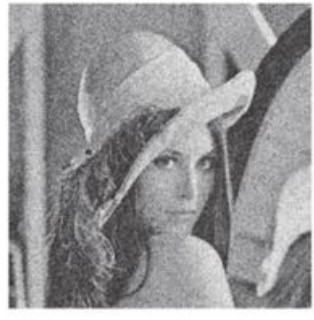

(c)

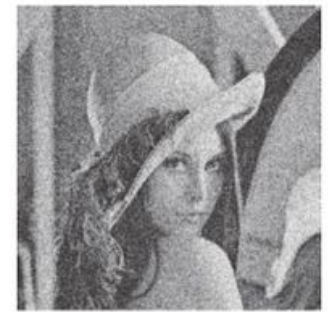

(d)

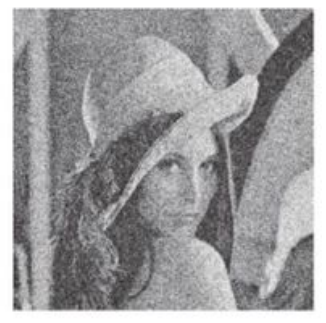

(e)

\section{Figure 6}

Lena image with different Gaussian noise:(a) without noise; (b) variance of 0.03 ; (c) variance of 0.04 ; (d) variance of 0.06 ; (e) variance of 0.08 . 


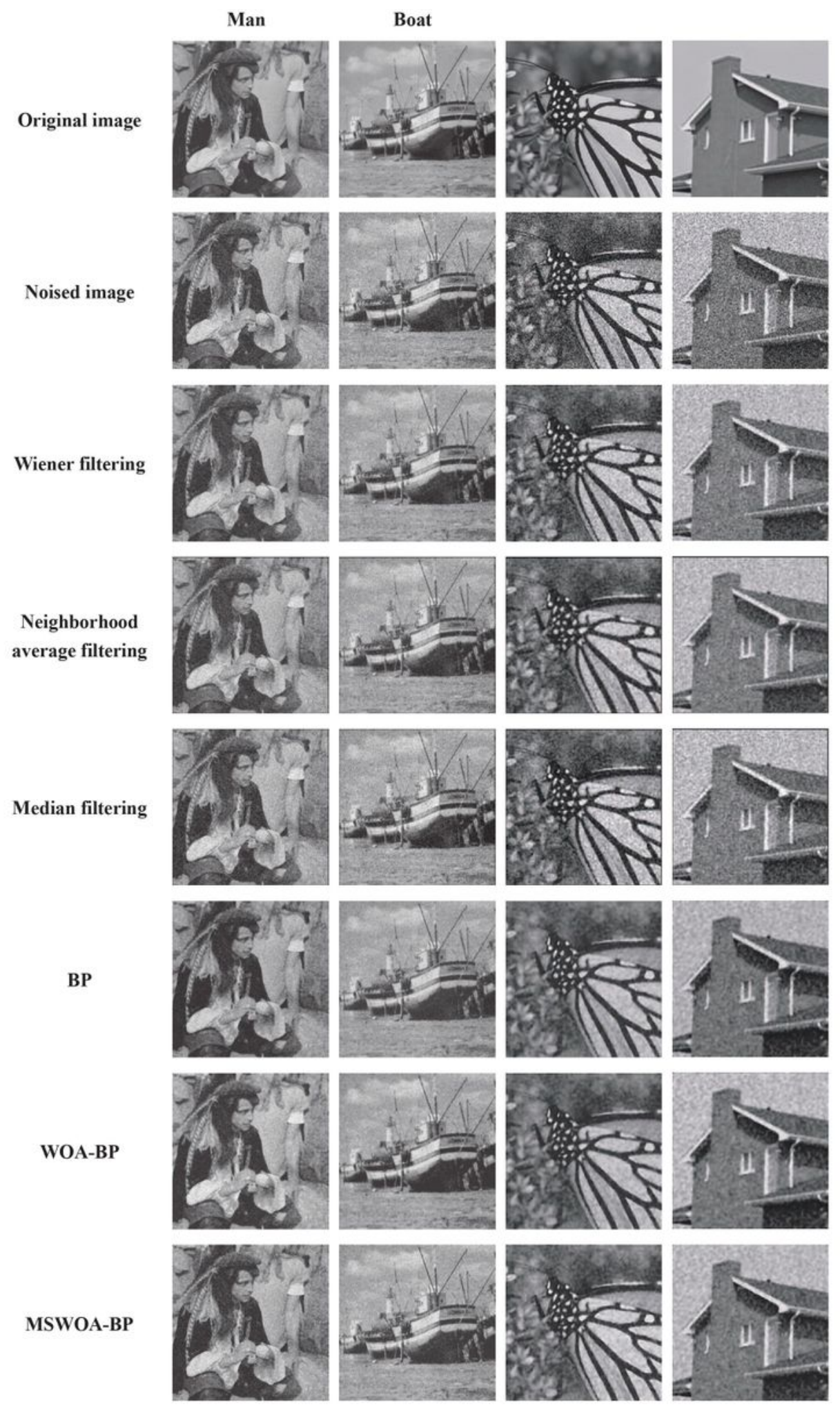

Figure 7

Denoising effect of different denoising algorithms for benchmark images 


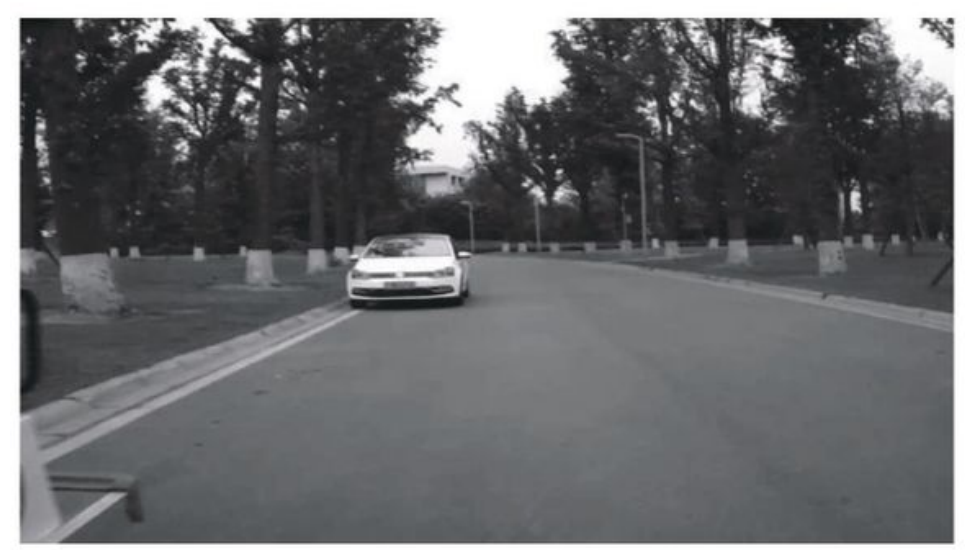

(a)

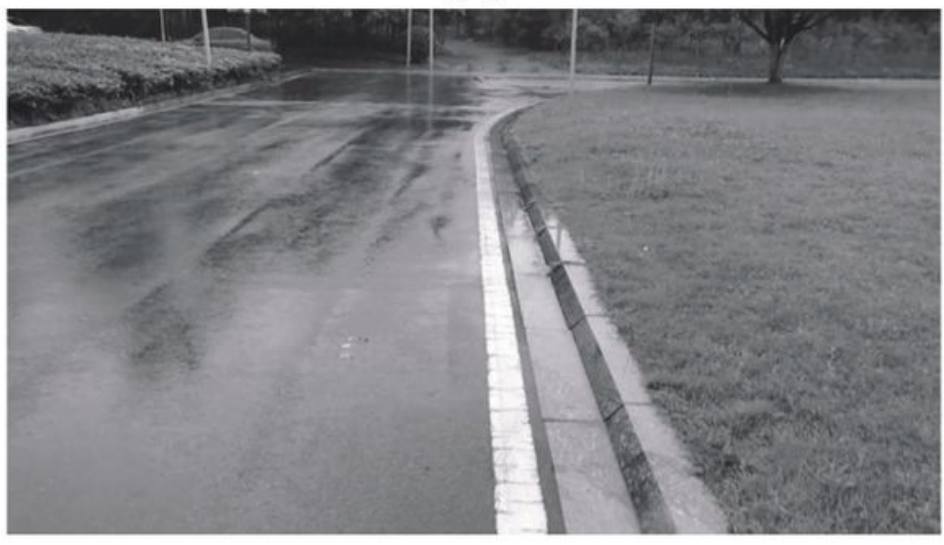

(c)

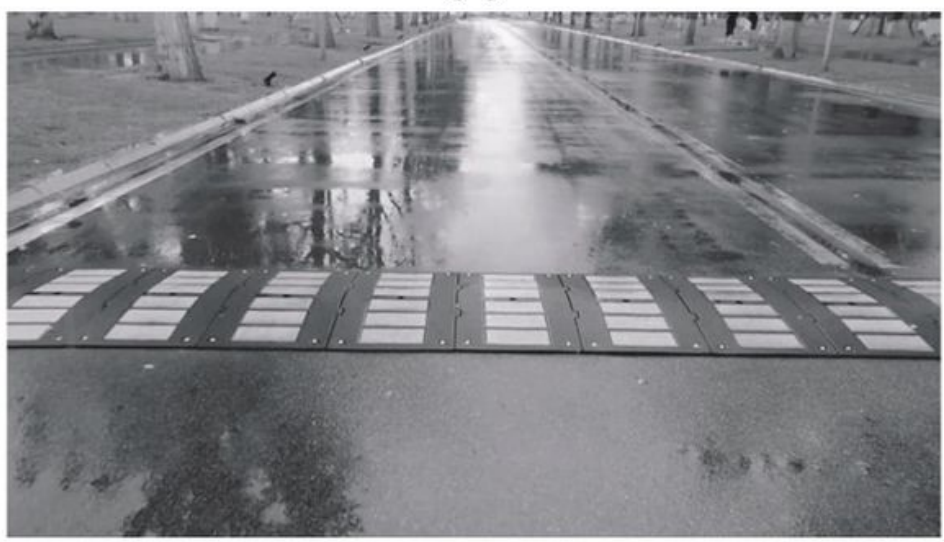

(e)

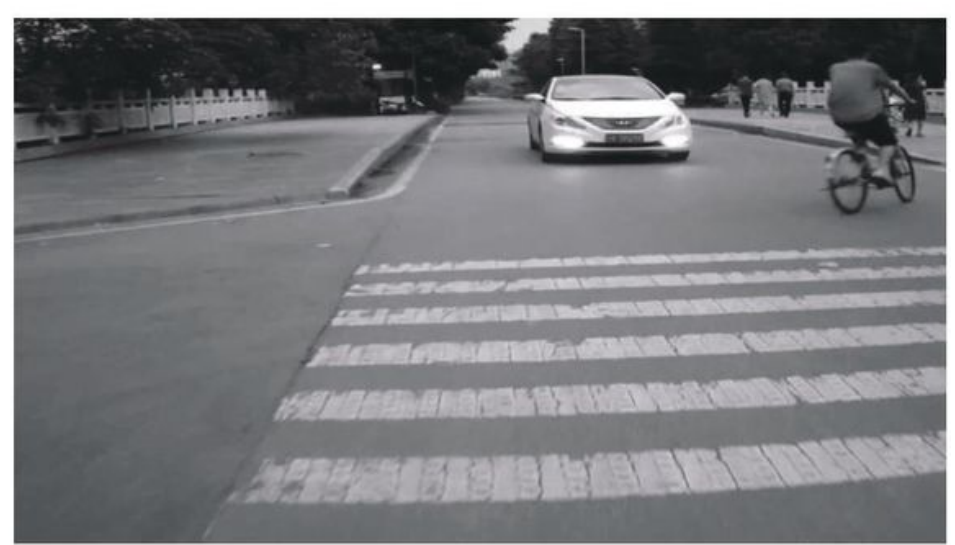

(b)

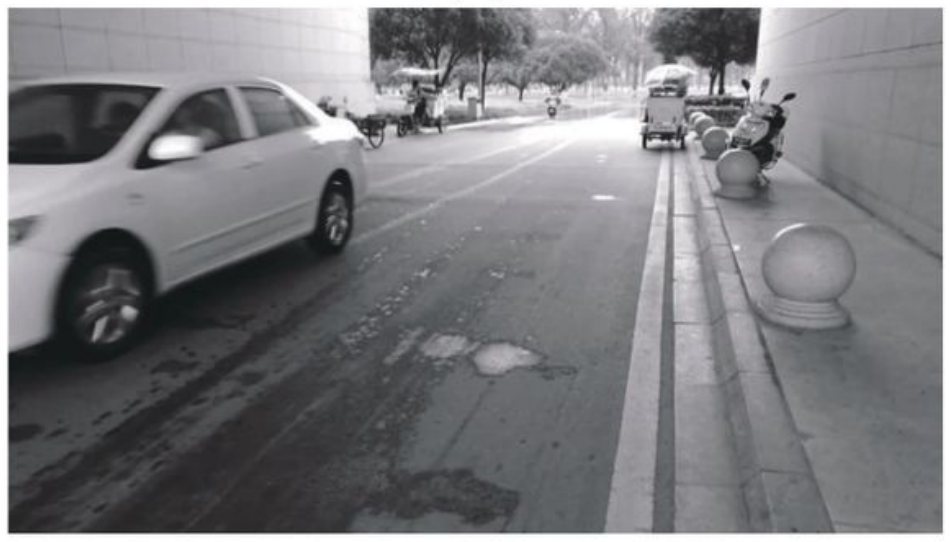

(d)

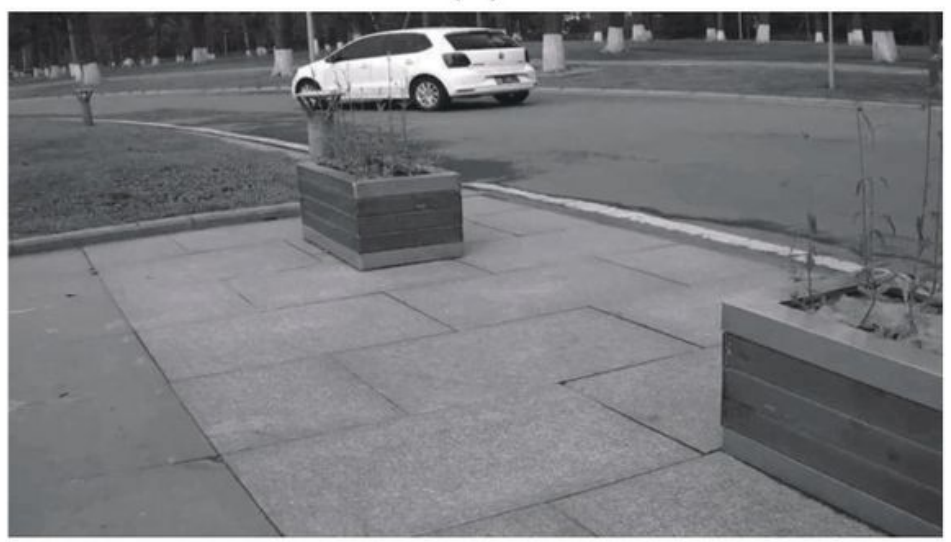

(f)

\section{Figure 8}

Original traffic images:(a) TI-1;(b) TI-2;(c) TI-3;(d) TI-4;(e) TI-5;(f) TI-6 


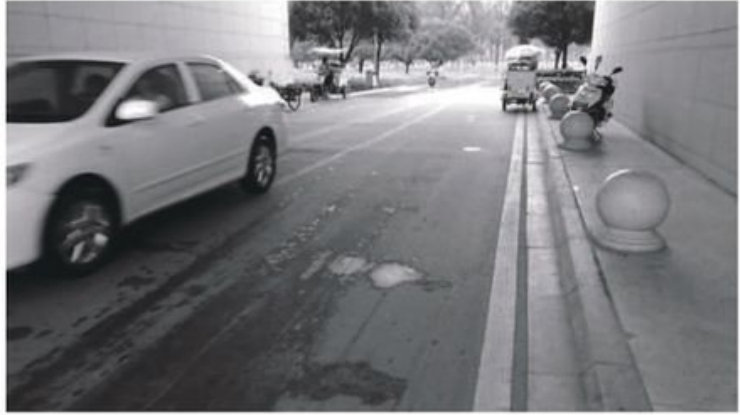

(a)

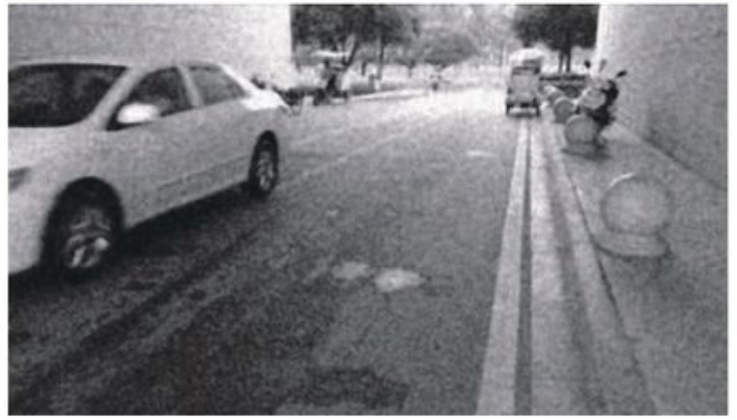

(c)

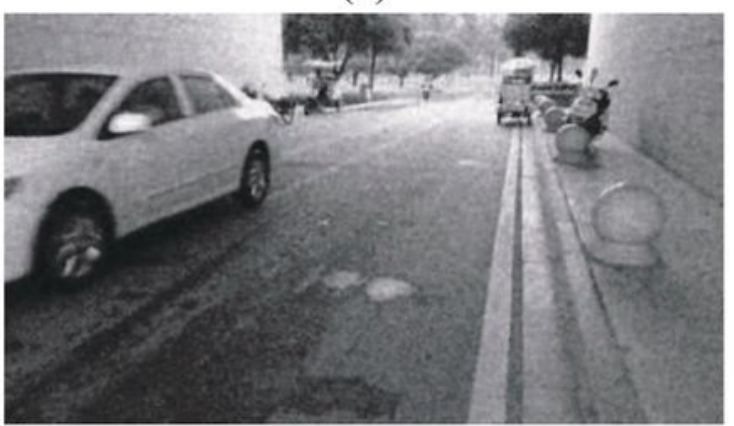

(e)

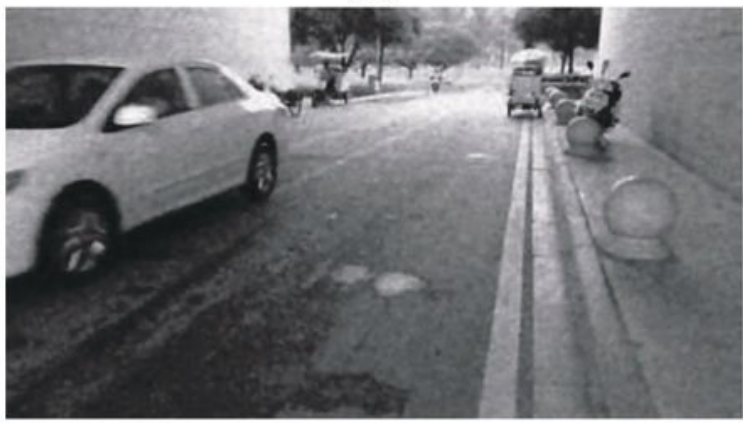

$(\mathrm{g})$

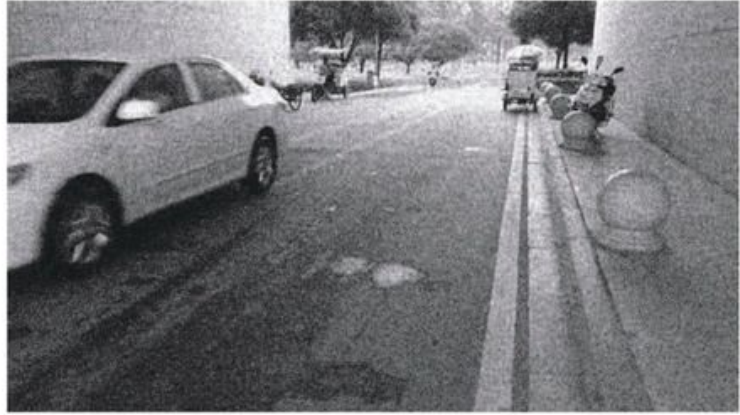

(b)

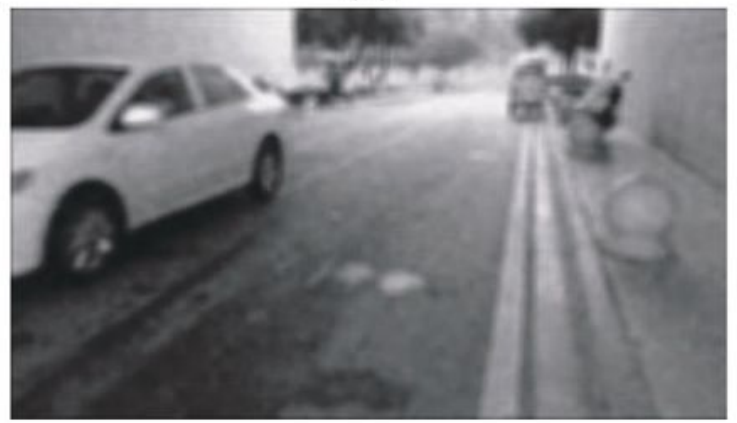

(d)

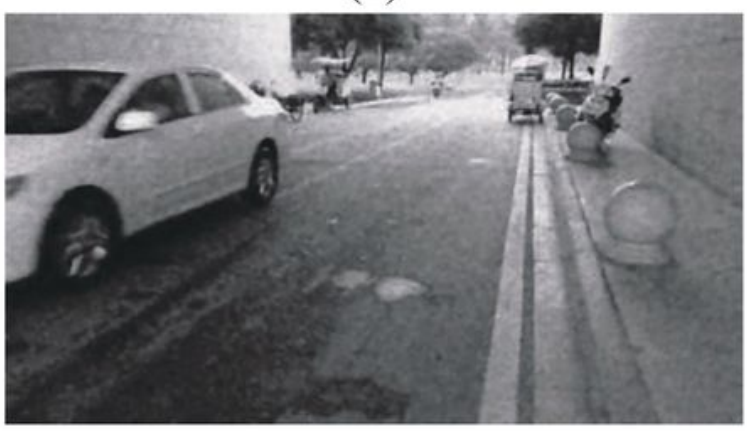

(f)

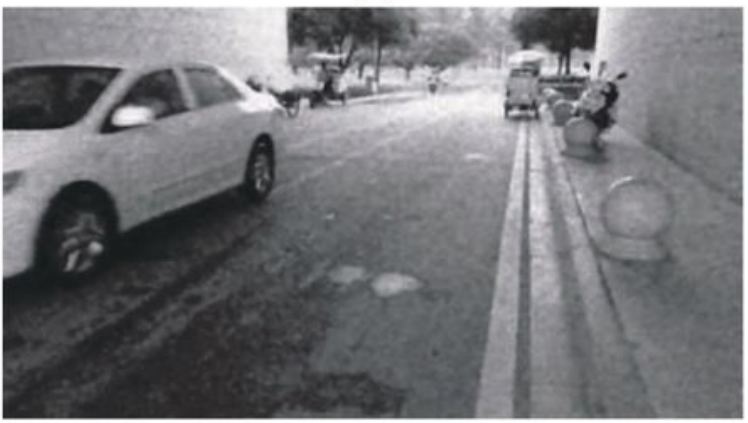

(h)

\section{Figure 9}

Denoising effect of different denoising algorithms for traffic images:(a) original image,(b) noised image, (c) Median flitering result,(d) Neighborhood average filtering result,(e) Wiener filtering result,(f) BP result, (g) WOA-BP result,(h) MSWOA-BP result 